Der effektive Diffusionskoeffizient wird also Null bei einer Temperatur von

$$
T=2 a(1-\imath) \frac{K}{R} .
$$

Nehmen wir $\alpha$ zu zwei Atomprozent, dann wird diese Nulltemperatur gleich $330^{\circ} \mathrm{C}$. Oberhalb dieser Temperatur kann also keine Komplexbildung mehr stattfinden. Wenn man die rohe Berechnung von $K$ berücksichtigt, ist die Übereinstimmung dieses Wertes mit der Erfahrung befriedigend.

Bei $300^{\circ} \mathrm{K}$ ergibt sich dann für $D^{\prime}$ ein Wert von

$$
D^{\prime}=D(1-1,64)=-D \cdot 0,64 .
$$

Für $D$ erhält man aus den Angaben bei $\mathrm{S}$ e i t $\mathrm{h}^{7}$ für Zimmertemperatur extrapoliert einen Wert von etwa $10^{-14} \mathrm{~cm}^{2} /$ Tag. Für $l$, das ist der mittlere Abstand zwischen den ursprünglich gelösten Atomen und den 1939.

7 W. S e it h, Diffusion in Metallen, Springer, Berlin
Komplexen, kann man etwa drei Atomabstände, also etwa $10^{-7} \mathrm{~cm}$, einsetzen. Wenn $n_{1}$ klein ist gegen $n_{2}$, fallen diese Größen heraus, und man erhält für die mittlere Wachstumszeit bei Zimmertemperatur einen Wert von etwa 4 Tagen, also auch hier eine größenordnungsmäßig sehr befriedigende Ưbereinstimmung. Genauere Rechnungen sind in Vorbereitung.

Es ist zu betonen, daß diese negative Diffusion in jedem übersättigten Mischkristall auftreten muß. Durch die neuen Versuche von Auer sowie von Köster, Steinert und Braumann ${ }^{8}$ scheint experimentell die Herstellung des reinen Falles, d. h. die Trennung von der bei höheren Temperaturen sich überlagernden Keimbildung, besonders gut gelungen zu sein. Zur Unterscheidung von der Keimbildung ist dabei, außer auf das metastabile Gleichgewicht, vor allem auf den linearen Verlauf der kinetischen Kurven bei Beginn des Vorgangs zu achten.

$8 \mathrm{Vgl}$. W. Köster, H. Steinert u. M. B rau m a n n, Z. Metallkde. 1951 im Druck.

\title{
Das amorphe Arsen
}

\author{
Von H. Richter und G. Breitling
}

Aus dem Röntgeninstitut der Technischen Hochschule Stuttgart

und dem Institut für Metallphysik am Max-Planck-Institut für Metallforschung, Stuttgart

(Z. Naturforschg. 6 a,721-741 [1951]; eingegangen am 28. Juli 1951)

\section{Erich Regener zum 70. Geburtstag}

Durch Aufdampfen und chemische Fällung wurden verschiedene amorphe As-Präparate hergestellt. Die an der Streukurve anzubringenden Korrektionen werden zusammengestellt und eingehend diskutiert. Von 12 Präparaten verschiedener Herstellung wurde durch FourierAnalyse die Atomverteilung bestimmt. Die hierbei erhaltenen Perioden, Atomzahlen und Abstandswerte sind in zwei Tabellen zusammengetragen. Die Betrachtung der bevorzugten Atomabstände gestattet weiterreichende Aussagen über den Aufbau des amorphen Körpers, als es bisher mit den Atomzahlen möglich war.

Man findet, daß im amorphen As nicht nur der Gitterbaustein erhalten geblieben ist, sondern die ganze Kette des As-Gitters (statistische Streuung der Atomlagen zugelassen). Bei As sind verschiedene Grade des amorphen Zustandes zu unterscheiden; ihnen allen fehlt die normale Raumgitterstruktur. In den sehr dünnen Aufdampfschichten $\left(D \approx 10^{-5} \mathrm{~cm}\right)$ liegt ein räumliches Gebilde miteinander verknüpfter As-Ketten vor und in den dicken Schichten $\left(D>10^{-3} \mathrm{~cm}\right)$ durch Parallelorientierung der Ketten eine Art von Stäbchenstruktur. Beim chemisch gefällten As wurde vorwiegend eine dem Gitter ähnliche Schichtbildung beobachtet. Der Übergang: amorph $\rightarrow$ kristallin zeichnet sich auch in der Kurve für die Lagenwahrscheinlichkeit der Atome ab. Das angegebene Kettenmodell erklärt in sehr befriedigender Weise die verschiedenen Erscheinungsformen des amorphen As.

$\mathrm{R}$ öntgenographische Untersuchungen an Ge von F ürst, Glocker und Richter ${ }^{1}$ sowie von Richter und $\mathrm{Fürst}{ }^{2}$ haben gezeigt, daß die Atomabstände im amorphen Zustande für die 1. und

1 O. F ürst, R. G lo cker u. H. Ri ch ter, Z. Naturforschg. 4 a, 541 [1949].
2. Koordination praktisch die gleichen sind wie im zugehörigen Raumgitter. Diese Abstände treten im kristallinen Ge als Zentralabstand und Kante des regulären Tetraeders, des Bauelementes des Ge-Gitters

2 H. Richter u. O. F ürst, Z. Naturforschg. 6a, 38 [1951]. 
auf. Das reguläre Tetraeder des Ge-Gitters kann daher auch für die amorphe Phase als Baueinheit dienen, zumal in beiden Zuständen auch die Atomzahl für die 1. Koordination die gleiche ist. Will man die Ergebnisse der Untersuchungen an sublimiertem amorphem As von Geiling und Richter ${ }^{3}$ in gleicher Weise deuten, so zeigt sich, daß hier die kürzesten Atomabstände praktisch mit den Abstandswerten des Bausteines des As-Gitters, eines einfachen (nicht zentrierten) Tetraeders ${ }^{4}$ übereinstimmen. Starke Unterschiede im strukturellen Aufbau von kristallinem und amorphem As bestehen dagegen zwischen den beobachteten Atomzahlen für die 2. und die höheren Koordinationen. So scheint es, als ob im amorphen As eine vom Gitter grundsätzlich verschiedene Bausteinverkettung vorliegen würde.

Unter diesen Umständen war es angezeigt, die Untersuchungen an sublimiertem As zu wiederholen und die Fourier-Analyse der Streukurven auch auf das chemisch gefällte As auszudehnen. Dabei war es wichtig, nur reinste Ausgangssubstanzen für die Präparatherstellung zu verwenden, bei den chemischen Fällungen jede störende Abscheidung von vornherein auszuschließen und auf die Dichtebestimmung größten Wert zu legen, da die Kenntnis der Dichte des untersuchten Stoffes für die genaue Bestimmung der Koordinationszahlen von allergrößter Bedeutung ist. Die Fourier-Analyse der Streukurve von feinkristallinen oder nichtkristallinen Stoffen gestattet lediglich die Bestimmung der Atomabstände und Koordinationszahlen, sie erlaubt dagegen keine Aussage über die Bindungsverhältnisse der Atome im Bauelement, insbesondere über die möglichen Winkelwerte. Die Kenntnis des Atomabstandes und der Atomzahl in der 1. Koordination ist daher von großer Wichtigkeit, da diese Größen allein für die Form und die Abmessungen des Bauelementes bestimmend sind.

I. Herstellung der amorphen As-Präparate durch Sublimation und chemische Fällung

1. Verdampfen von As im Quarz-oder Glasrohr

Um möglichst reines As für die Sublimation zu erhalten, wurde das vorliegende kristalline As mehrfach im Hochvakuum bei ungefähr $10^{--5} \mathrm{~mm} \mathrm{Hg}$ einige Stdn. auf etwa $200^{\circ} \mathrm{C}$ erhitzt. Durch eine solche Glühbehand-

${ }^{3}$ S. Geiling u. H. Richter, Acta Cryst. 2, 305 [1949].

4 Im Gegensatz zum „regulären“ Tetraeder ist hier mit "Tetraeder" schlechthin das gleichschenklige Tetraeder bezeichnet. lung wird die metallische Ausgangssubstanz praktisch vollkommen von dem bei ungefähr $150^{\circ} \mathrm{C}$ sublimierenden $\mathrm{As}_{2} \mathrm{O}_{3}$ gereinigt.

Zur Herstellung dickerer Sublimationsschichten wurde ein Quarzrohr von $50 \mathrm{~cm}$ Länge und $3 \mathrm{~cm}$ lichter Weite verwendet. Der untere Teil dieses Rohres mit dem zu verdampfenden metallischen As ragte etwa $5-10 \mathrm{~cm}$ tief in einen elektrischen Ofen hinein, der obere Teil des Sublimationsrohres, in dem sich das sublimierte As niederschlug, war mittels einer Asbestplatte gegen eine eventuelle Erwärmung durch den Ofen geschützt. Auch bei diesen Sublimationsversuchen im Quarz- oder Glasrohr lag der Gasdruck um $10^{-5} \mathrm{~mm} \mathrm{Hg}$.

Mit Beginnn der Sublimation, bei etwa $200^{\circ} \mathrm{C}$, entsteht zunächst ein gelbbrauner Niederschlag, der mit zunehmender Schichtdicke eine mattschwarze Färbung annimmt. Diese sehr dünnen und zusammenhängenden amorphen Aufdampfschichten ändern während des weiteren Aufdampfens mehrfach ihr Aussehen. Mit wachsender Schichtdicke nehmen sie zunächst stellenweise (schlagartig) einen metallischen Glanz an. Unmittelbar danach tritt in diesen glänzenden Bezirken entsprechend Abb. $1^{*}$ eine strahlenartige Rißbildung auf. Bei weiterer Sublimation geht die Linienstruktur der Abb. 1 allmählich verloren, und es bildet sich eine stäbchenförmige Struktur senkrecht zur Glaswand aus (vgl.Abb. 2). Diese Sublimationsschichten bestehen aus einzelnen Stäbchen, die sich aus ihrem Verband leicht herauslösen lassen. Die Stäbchen selbst zeigen keine Spaltbarkeit, weder in ihrer Längs- noch Querrichtung. Diese Aufdampfschichten wurden entweder als dicke massive Blöcke in Reflexion untersucht, meistens aber als dünne Folien mit einer ungefähren Dicke $D \approx 0,2 \mathrm{~mm}$ durchstrahlt. Pyknometrische Dichtemessungen ergaben für das sublimierte As mit Stäbchenstruktur unabhängig von seiner Schichtdicke den Wert $\sigma=4,70 \mathrm{~g} / \mathrm{cm}^{3}$.

Die schlagartige Veränderung im Aussehen der sehr dünnen, mattschwarzen Sublimationsschichten tritt beim Erreichen einer bestimmten Schichtdicke auf. Kühlt man nämlich das Quarzrohr mittels einer weitspiraligen Kühlschlange, so schlägt sich das sublimierte As vornehmlich an den stark gekühlten Stellen des Quarzrohres nieder. An diesen Stellen ist im Verlauf der Sublimation das veränderte Aussehen der Aufdampfschicht zuerst zu beobachten.

2. Verdampfen von As im Wo-Schiffchen der Aufdampfapparatur

Weiter wurden durch Verdampfen von As aus einem Wo-Schiffchen der Aufdampfapparatur amorphe Schichten hergestellt. Hierbei wurde As in sehr gutem Hochvakuum

* Abb. 1 und 2, s. Tafel S. 724 b. 
verdampft und bei Zimmertemperatur auf Steinsalz niedergeschlagen. Um die Diffusion von Fremdstoffen in den Dampfstrom des sublimierenden As möglichst auszuschlieBen, wurde der Abstand zwischen Wo-Schiffchen und Steinsalzplättchen klein gehalten und nach $\mathrm{W}$ alke nh orst ${ }^{5}$ die Aufdampfgeschwindigkeit (hohe Aufdampftemperatur) groß gewählt. In einer anderen Meßreihe wurde $A s$ in Gegenwart von Fremdstoffen (Öldämpfen) bei großem Abstand zwischen Wo-Schiffchen und Auffangfläche langsam verdampft und auf einer nach $\mathrm{Ri} \mathrm{ch}$ t e r und B e r ck h e me ${ }^{6}{ }^{6}$ mit flüssiger Luft gekühlten Steinsalzspaltfläche niedergeschlagen. Im Gegensatz zur ersten Aufdampfschicht sind hier Fremdatome in die amorphe As-Schicht mit eingebaut.

Die zum Teil noch durchscheinenden Schichten auf Steinsalz als Träger wurden über Wasser abgelöst ${ }^{7}$ und auf einem geeigneten Präparathalter aufgefangen. Da die Belichtungszeiten bei Durchstrahlung einer einzigen Aufdampfschicht, deren durchschnittliche Dicke ${ }^{8}$ bei etwa $D=2,5 \cdot 10^{-5} \mathrm{~cm} \mathrm{lag}$, außergewöhnlich groß wären, wurden etwa 20 bis 30 solcher Schichten übereinander gepackt. Hierdurch wird der Streuanteil der sehr dünnen Kollodiumschicht, die als Träger für die ersten aufgefangenen As-Schichten dient, mit wachsender Schichtzahl praktisch unmerklich.

\section{Reduktion von $\mathrm{As}_{2} \mathrm{O}_{3}$ mit $\mathrm{SnCl}_{2}$}

Das in konzentrierter $\mathrm{HCl}$ gelöste $\mathrm{As}_{2} \mathrm{O}_{3}$ wurde durch Zusetzen von salzsaurer $\mathrm{SnCl}_{2}$-Lösung zu As reduziert. Die Reduktion wurde in stark salzsaurer Lösung vorgenommen, um ein Ausfallen von $\mathrm{As}_{2} \mathrm{O}_{3}$ und Zinnsäure zu verhindern. Aus demselben Grunde wurde das gefällte As anfangs mit konzentrierter $\mathrm{HCl}$ und erst am Schluß mit dest. Wasser ausgewaschen. Das aus der konzentrierten $\mathrm{As}_{2} \mathrm{O}_{3}$-Lösung gefällte $\mathrm{As}$ sah unabhängig von der Fällungstemperatur $\left(T \approx 20^{\circ} \mathrm{C}\right.$ und $\left.T \approx-40^{\circ} \mathrm{C}\right)$ anfangs dunkelbraun aus; seine Farbe ging allmählich in ein tiefes Schwarz über. Pyknometrische Dichtemessungen ergaben für das mit $\mathrm{SnCl}_{2}$ gefällte As $\sigma=3,64 \mathrm{~g} / \mathrm{cm}^{3}$.

\section{Reduktion von $\mathrm{As}_{2} \mathrm{O}_{3}$ mit $\mathrm{H}_{3} \mathrm{PO}_{2}$}

Das in konzentrierter $\mathrm{HCl}$ gelöste $\mathrm{As}_{2} \mathrm{O}_{3}$ wurde durch Zusetzen von $\mathrm{H}_{3} \mathrm{PO}_{2}$ zu As reduziert. Auch hier wurde das gefällte As zur Vermeidung des Ausfallens von $\mathrm{As}_{2} \mathrm{O}_{3}$ mit $\mathrm{HCl}$ abnehmender Konzentration ausgewaschen. Es sah je nach der Fällungstemperatur rotbraun $(T \approx$ $\left.-15^{\circ} \mathrm{C}\right)$ oder tiefschwarz $\left(T \approx 20^{\circ} \mathrm{C}\right)$ aus. Pyknometrische Dichtemessungen lieferten $\sigma=3,66 \mathrm{~g} / \mathrm{cm}^{3}$, in guter Ubereinstimmung mit dem oben gefundenen Wert.

5 W. Walkenhorst (Diss. Hannover 1940) beobachtete bei langsamem Aufdampfen von Al durch Einbau von Fremdgasen eine Dichteverringerung bis zu $30 \%$.

6 H. R i ch t er u. H. B e r c k h e m er, "Struktur des amorphen Antimon.“ Die Arbeit erscheint demnächst.

7 Die dünnen, mattschwarzen As-Schichten $\left(D \approx 10^{-5} \mathrm{~cm}\right)$ besitzen eine weitgehend zusammenhängende Struktur, die dickeren Schichten zeigen dagegen eine ausgesprochene Linien- und Stäbchenstruktur (vgl. Abb. 1 und 2), wodurch der anfängliche innere Zusammenhang der Aufdampfschichten besonders beim Fehlen von Fremdatomen verlorengeht.
Bei der Herstellung dieser As-Präparate wurde von $\mathrm{As}_{2} \mathrm{O}_{3}$-Lösungen verschiedener Konzentration ausgegangen und in verschiedenen Etappen gefällt, um eine eventuelle Abhängigkeit der Atomanordnung im ausgefallenen As von der Konzentration der Ausgangslösung bzw. der Reaktionsgeschwindigkeit zu prüfen. Zur Untersuchung eines vielleicht vorhandenen Einflusses der Fällungstemperatur auf die Atomanordnung des amorphen As wurde sowohl bei Zimmertemperatur als auch bei tiefer Temperatur gefällt (langsames Zusetzen einer kleinen Menge des gekühlten Reduktionsmittels bei gleichzeitiger Kühlung der $\mathrm{As}_{2} \mathrm{O}_{3}$-Lösung). Um eine Oxydation des reduzierten As zu verhindern, wurden die Fällungsprodukte mit Alkohol und Äther versetzt und im Vakuum getrocknet. Diese pulverförmigen As-Präparate wurden für die röntgenographische Untersuchung zwischen zwei ebenen Metallplatten mit Handdruck zu dünnen durchstrahlungsfähigen Plättchen mit einer Dicke $D \approx 0,2 \mathrm{~mm}$ oder aber zu dicken, strahlenundurchlässigen Blöcken gepreßt.

\section{A u f n a hmetechnik}

\section{Technische Einzelheiten}

Sämtliche Aufnahmen wurden mit monochromatischer $\mathrm{Cu}-\mathrm{K}_{a}-$ Strahlung $\left(\lambda_{\mathrm{Cu}-\mathrm{K}_{a}}=1,54 \AA\right)$ gemacht. Aufnahmen mit Mo-K ${ }_{a}$-Strahlung $\left(\lambda_{\mathrm{Mo}-\mathrm{K}_{a}}=0,71 \AA\right)$ sind wegen der störenden K-Eigenstrahlung von As $\left(\lambda_{\mathrm{K}-\mathrm{Abs}}=1,042 \AA\right)$ praktisch nicht möglich. Die Monochromasie der Strahlung wurde durch Braggsche Kristallreflexion an Pentaerythrit oder an Steinsalz erreicht. Zur Aufnahme diente eine Debye-Kammer von 81,4 mm Durchmesser. Als Blenden wurden Schlitzblenden von $50 \mathrm{~mm}$ Länge und vom Querschnitt $0,2 \times 2 \mathrm{~mm}^{2}$ verwendet, so daß näherungsweise mit einem parallelen Strahlenbündel gearbeitet wurde. Die Benützung von Schlitzblenden ermöglichte eine weitgehende Herabsetzung der Belichtungszeiten. Um die Luftstreuung auszuschalten, wurde die Kammer evakuiert, so daß der Gasdruck im Durchschnitt 2-3 mm Hg betrug. Durch Absorptionsversuche mittels Al-Folien von 15 bis $100 \mu$ Dicke konnte gezeigt werden, daß auch bei gefälltem As nur die L-Eigenstrahlung von $\mathrm{As}\left(\lambda_{\mathrm{L}}\right.$ I Abs. $\left.\approx 8,5 \AA\right)$ als störende Streustrahlung mit merklicher Intensität auftrat, d. h. daß bei der Reduktion mit $\mathrm{SnCl}_{2}$ keine Zinnsäure ausgefallen war. So genügten bereits Al-Folien von $25 \mu$ Dicke, um die störende L-Strahlung von As praktisch auszuschalten. Die amorphen As-Präparate wurden entweder nach $\mathrm{Richter}$ und $\mathrm{F}$ ürst ${ }^{2}$ und Menzer ${ }^{9}$ als dünne Schichten durchstrahlt oder aber nach $\mathrm{D}$ e b y e und Menke10, Glocker und Hendus ${ }^{11}$ sowie $\mathrm{H}$ endus ${ }^{12}$ als massive Blöcke in Reflexion untersucht. Für die Aufnahmen wurde Agfa-Laue-Röntgenfilm ver-

8 Die Schichtdicke wurde aus der Aufnahme selbst bestimmt (vgl. III, 3) oder aus der Lichtdurchlässigkeit geschätzt.

9 G. M e n z e r, Z. Naturforschg. 2a, 335 [1947].

10 P. D e b y e u. H. M e n k e, Ergebn. techn. Röntgenkunde 2, 16 [1931]; H. Menke, Physik. Z. 33, 593 [1932].

11 R. G locker u. H. H end us, Z. Elektrochem. angew. physik. Chem. 48, 327 [1942].

12 H. H e n d u s, Z. Physik 119, 265 [1942]. 
wendet. Die Belichtungszeiten betrugen im Mittel 50 Stdn. bei $40 \mathrm{kV}$ und $20 \mathrm{~mA}$. Die Aufnahmen wurden mit dem lichtelektrischen Registrier-Photometer von Zeiß photometriert und die Photometerkurven mittels Schwärzungstreppen auf relative Intensitäten umgezeichnet.

\section{Präparatform und Präparatanordnung}

Die beobachtete Intensitätsverteilung ist u. a. auf Absorption der Strahlung im Präparat zu korrigieren; dies bedingt die Verwendung solcher Präparatformen, die eine leichte Erfassung der Absorptionskorrektion gestatten. Unter den möglichen Präparatformen zeichnet sich das plattenförmige Präparat durch seine leichte Herstellbarkeit und durch die große Übersichtlichkeit der Absorptionsverhältnisse aus.

Die bei gefälltem As beobachtete Streuintensität in unmittelbarer Nachbarschaft des Nullpunktes (Kleinwinkelstreuung) läßt auf das Vorliegen sehr kleiner Partikelchen schließen (vgl. Abb. $7^{*}$ ). Es wurde daher auch auf die experimentelle Erfassung dieser Nullpunktstreuung großer Wert gelegt. Bei Verwendung unendlich dicker Plattenpräparate kommen hierfür nur extrem kleine Einstrahlwinkel in Betracht, da sonst eine völlige Absorption der Kleinwinkelstreuung erfolgt. Leider ist mit kleiner werdendem Einstrahlwinkel $\alpha$ eine Vergrößerung des bestrahlten Volumens verbunden, was für Winkelbereiche um $2 \vartheta=90^{\circ}$ zu einer starken geometrischen Verbreiterung der Interferenzen führt, entsprechend der Größe der Projektion des bestrahlten Volumens auf dem Film. Dem Übergang zu kleinen Einstellwinkeln sind also Grenzen gesetzt.

Um diese Schwierigkeiten auszuschließen, wurde zu dünnen, strahlendurchlässigen Präparaten bei Einstrahlwinkeln $\alpha=20^{\circ}$ bis $40^{\circ}$ übergegangen. Diese Präparatanordnung hat folgende Vorteile:

a) Durch Zusammensetzen der Streukurve aus der Reflexions- und Durchstrahlungsseite der Aufnahme erhält man den Intensitätsverlauf für den gesamten Winkelbereich, also auch für die nächste Umgebung des Nullpunktes, während bei den undurchlässigen Präparaten die Nachbarschaft des Nullpunktes nicht erfaßt werden kann.

b) Innerhalb der schraffierten Winkelbereiche der Abb. 3, welche für die Zusammensetzung der Streukurve herangezogen werden, sind die Absorptionskorrektionen nach Abb. 4 nahezu konstant. Hier sind die Korrektionskurven $Q_{\mathrm{D}}$ und $Q_{\mathrm{R}}$ für $\alpha=40^{\circ}$ und $\mu D=1$ gezeichnet. Die Punkte A und B bzw. C und D auf den Korrektionskurven $Q_{\mathrm{D}}$ bzw. $Q_{\mathrm{R}}$ geben dabei die Korrektionsfaktoren für die entsprechenden

* Abb. 7, s. Tafel S. 724 b.
Punkte der Abb. 3 auf der Durchstrahlungs- bzw. Reflexionsseite an. Weiter ist bei großem Einstrahlwinkel der Einfluß der Schwankungen von $\alpha$, die durch die Unebenheit der Präparate bedingt sind, auf ein Minimum reduziert.

c) Der mittlere Einstrahlwinkel $\alpha$ kann in erster Näherung unmittelbar der Aufnahme entnommen werden. Er zeichnet sich als scharfer Einschnitt in der Filmschwärzung ab (vgl. Abb. 7). Hierbei ist es von Vorteil, den Einstellwinkel $\alpha$ so zu wählen, daß dieser

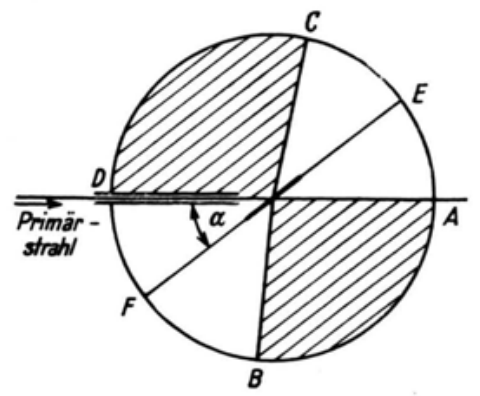

Abb. 3. Zusammensetzung der Streukurve aus den schraffierten Bereichen.

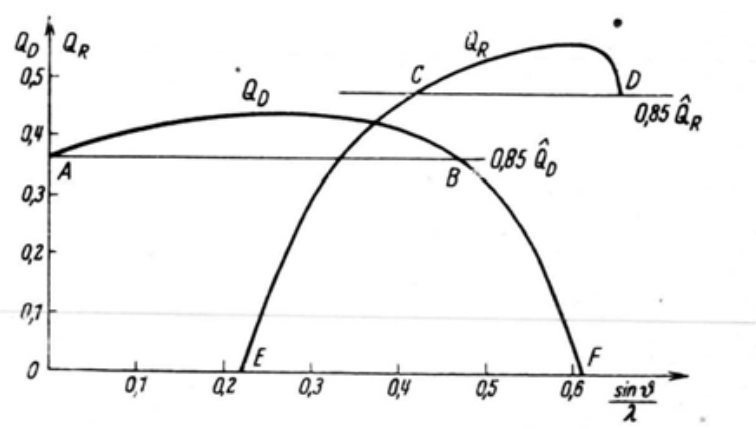

Abb. 4. Korrektionsfaktoren $Q_{\mathrm{D}}$ und $Q_{\mathrm{R}}$ als Funktion von $\sin \vartheta / \lambda$.

Einschnitt in ein Gebiet großer Schwärzung fällt. Die Einsatzstelle der Filmschwärzung hebt sich dann besonders deutlich ab.

d) Eine solche Aufnahme erlaubt ferner, die Schichtdicke $D$ bzw. die Größe $\mu D$ des durchstrahlten Präparates aus der Streuintensität einer in Reflexion und Durchstrahlung erhaltenen Interferenz zu berechnen.

e) Bei der hier gewählten Präparatform und -Anordnung ergibt sich die günstigste Filmschwärzung $(S=1,5$ bis 2,5$)$ für sämtliche Streuwinkel $2 \vartheta$ bei geeigneter Präparatdicke von selbst, da die großen $2 \vartheta$-Werte von der Reflexionsseite und die kleinen $2 \vartheta$-Werte von der Durchstrahlungsseite erfaßt werden.

f) Bei nicht völliger Homogenität der durchstrahlten Schicht besitzen die Beugungsdiagramme der Re- 
Abb. 5. Berührungsladung auf der Dielektrikum-Oberfläche, durch Bestäuben sichtbar gemacht $(\% / 10 \times$ natürl. Größe).

Abb. 6. Ladungsverteilung auf der Oberfläche des Dielektrikums nach Durchbruch der Luftschicht. $d=8 \mathrm{~mm}, U=$ $900 \mathrm{~V}$. Meßelektrode negativ $(1,8 \times$ natürl. Gr.).

Abb. 7. Vergrößertes Bild einer Oberflächenladung nach Durchbruch der Luftschicht. $d=2 \mathrm{~mm} ; U=10 \mathrm{kV} ; \mathrm{Me}$ elektrode positiv $(4,2 \times$ natürl. $\mathrm{Gr}$.).
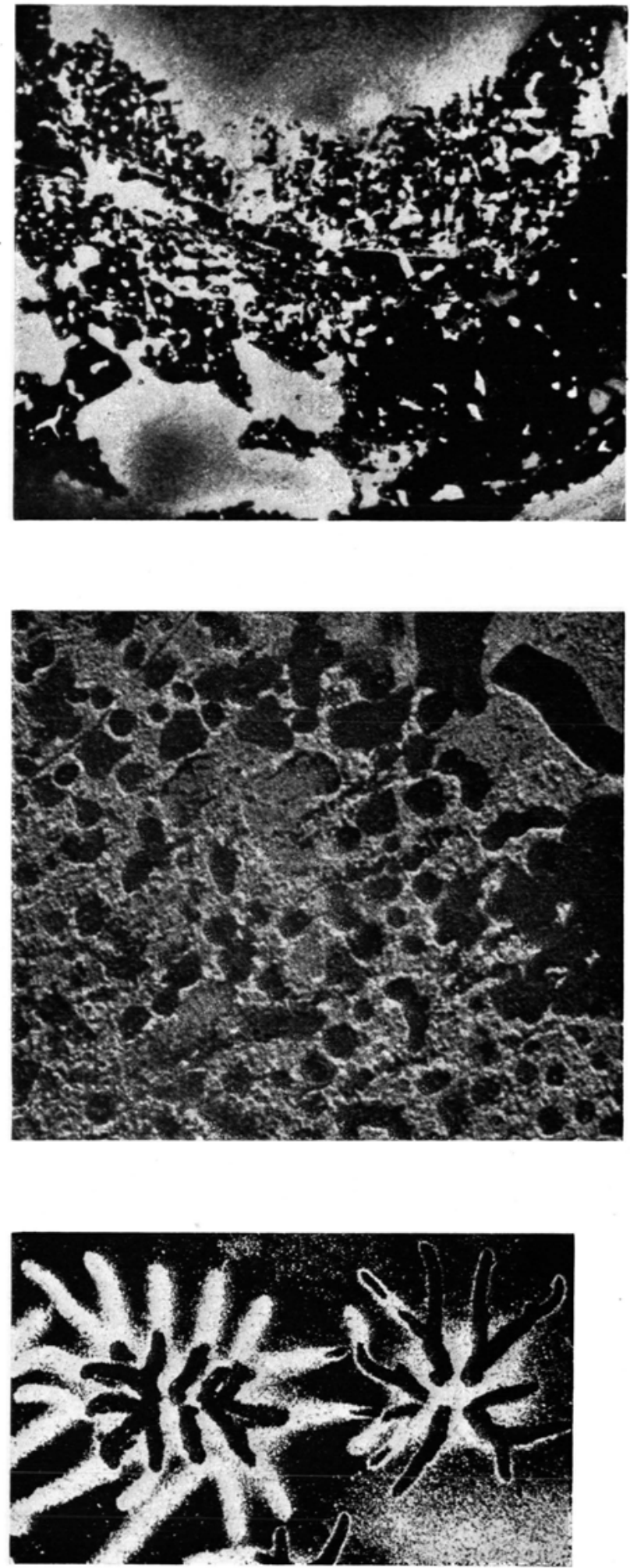


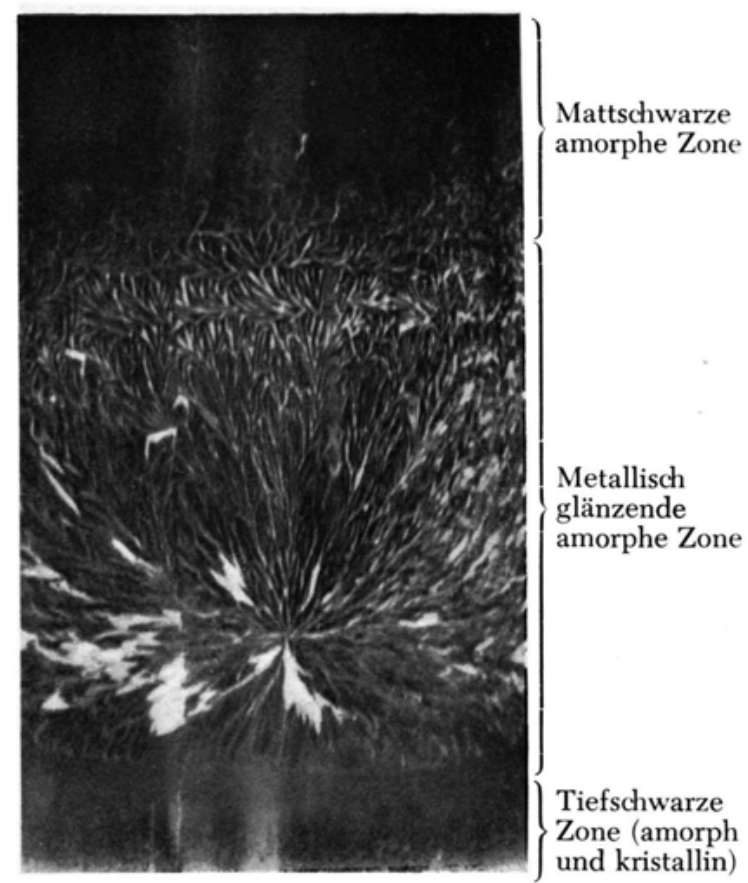

Abb. 1. Verschiedene Zonen einer dünnen Aufdampfschicht von As $\left(D \approx 10^{-4} \mathrm{~cm}\right.$, Vergr. 2).

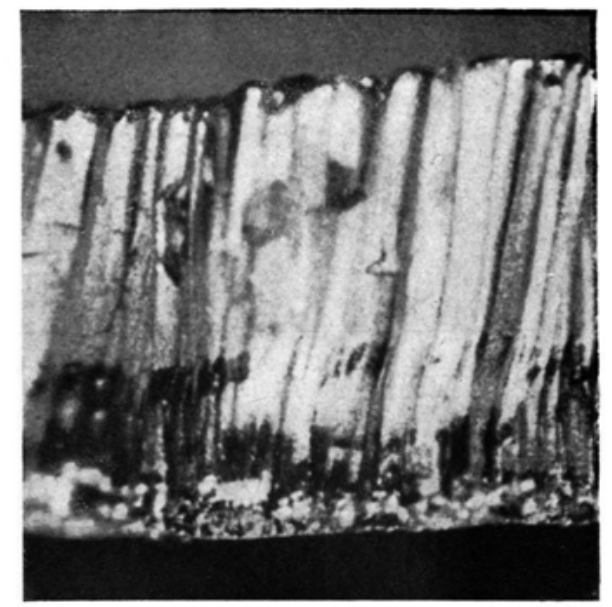

Abb. 2. Dicke Aufdampfschicht von As mit Stäbchenstruktur $\left(D \approx 7 \cdot 10^{-2} \mathrm{~cm}\right.$, Vergr. 75$)$.

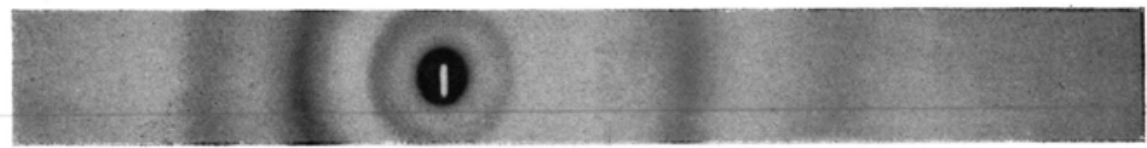

Abb. 7. As, amorph, gefällt mit $\mathrm{SnCl}_{2}, \mathrm{Cu}-\mathrm{K}_{\alpha}-$ Strahlung,

Einstellwinkel $\alpha=30^{\circ}$. (Links: Durchstrahlung, rechts: Reflexion.)

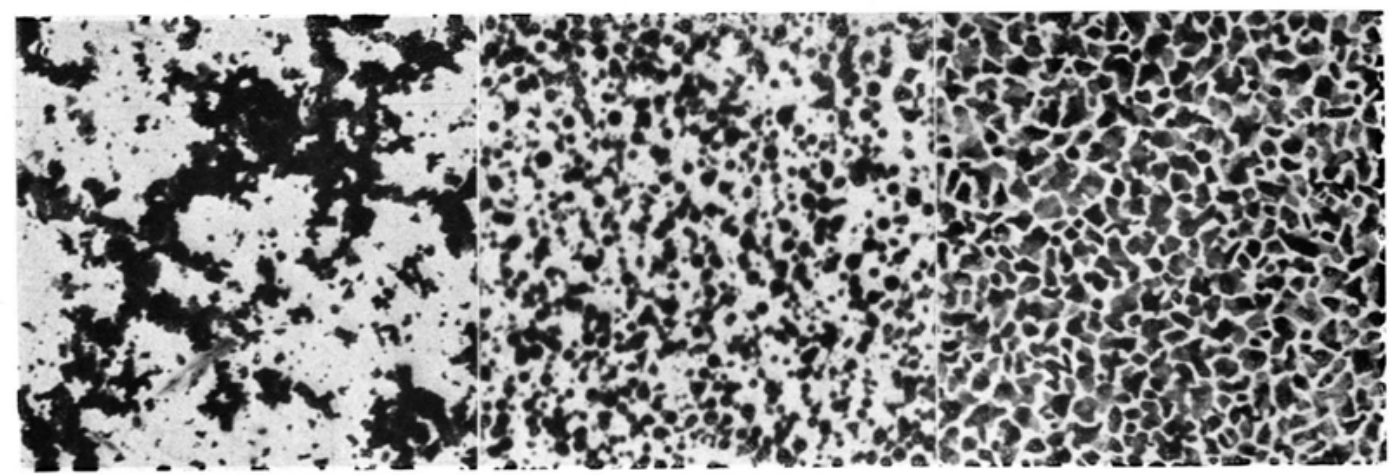

Abb. 11. As, amorph, gefällt (Vergr.: 25000).
Abb. 12. As, amorph, sublimiert (Vergr.: 25000).
Abb. 13. As, kristallin, umgewandelte Aufdampfschicht. 
flexions- und Durchstrahlungsseite ein verschiedenes Aussehen, so daß Unterschiede ${ }^{13}$ im inneren Aufbau der Schicht erkennbar werden.

\section{Auswertung der Aufnahmen}

\section{Exzentrizitäts-Korrektion}

An der experimentellen Streukurve sind verschiedene Korrektionen anzubringen. Da die Korrektionen rechnerisch als Funktion des Streuwinkels $2 \vartheta$ erhalten werden, ist zunächst der Zusammenhang zwischen dem Nullpunktsabstand $s$ einer Interferenz auf dem Film und dem Winkel $2 \vartheta$ zu ermitteln. Die Proportionalität zwischen dem Abstand $s$ und dem Streuwinkel $2 \vartheta$ ist nur bei genauer Zentrierung des Präparates erfüllt, was aber bei plattenförmigen Präparaten nur schwer zu erreichen ist. Die Exzentrizität des Präparates wurde, wie die folgenden Ausführungen zeigen, aus der Ringlage der amorphen Interferenzen errechnet, deren genaue Lage man zuvor aus Eichaufnahmen mit Gold bestimmt hatte.

a) Bei gegebenem Nullpunkt. In Abb. 5 bezeichnet $\mathrm{MP}=\varepsilon$ die Exzentrizität des Präparates. Nach dem Sinussatz ist:

$$
\varepsilon=\frac{\sin \left(2 \vartheta_{\mathrm{R}}-2 \vartheta_{\mathrm{R}}^{\prime}\right)}{\sin 2 \vartheta_{\mathrm{R}}} A
$$

mit $2 \vartheta$ bzw. $2 \vartheta^{\prime}$ als Streuwinkel bei Vorhandensein bzw. Fehlen von $\varepsilon$. Bei exakter Zentrierung gilt $\vartheta_{\mathrm{R}}{ }^{\prime}=$ $s_{\mathrm{R}} / 2 A$, wobei $s_{\mathrm{R}}$ den Abstand des Interferenzringes vom Nullpunkt $O$ (Durchstoßpunkt des Primärstrahles) und $A$ den Kammerradius bedeutet. Bei Substitution von $\vartheta_{\mathrm{R}}{ }^{\prime}$ folgt für die Exzentrizität

$$
\varepsilon=A \frac{\sin \left(2 i_{\mathrm{R}}-{ }_{\mathrm{R}} s\right)}{\sin 2 \vartheta_{\mathrm{R}}} .
$$

Diese Beziehung gilt nur bei bekannter Lage des Nullpunktes O.

Bei den dünnen, strahlendurchlässigen Plattenpräparaten ist die Größe der Absorption im Gegensatz zum zylindrischen Präparat für Punkte, die symmetrisch zum Durchstoßpunkt $\mathrm{O}$ des Primärstrahls liegen, im allgemeinen verschieden. Hierdurch erfolgt, insbesondere in der Nähe von Schattengrenzen, eine unterschiedliche Verschiebung der Maxima. Die Bestimmung des Nullpunktes $\mathrm{O}$ durch bloßes Halbieren

13 Mitunter, vor allem bei amorphem Sb, waren die Streubilder der Reflexionsseite infolge beginnender Oberflächenkristallisation verschieden von denen der Durchstrahlungsseite. der Strecke zwischen den entsprechenden Maxima bzw. Minima beiderseits des Nullpunktes $\mathrm{O}$ ist daher nur dann erlaubt, wenn die Absorption für die Umgebung der betrachteten Punkte in erster Näherung konstant ist oder aber dieselbe Änderung erfährt. Im anderen Falle, ebenso auch bei den völlig undurchlässigen Präparaten, ist wie folgt vorzugehen.

b) Bei fehlendem Nullpunkt. Die Bestimmung der Präparatexzentrizität ist in diesem Fall nur möglich, wenn die genaue Lage von mindestens
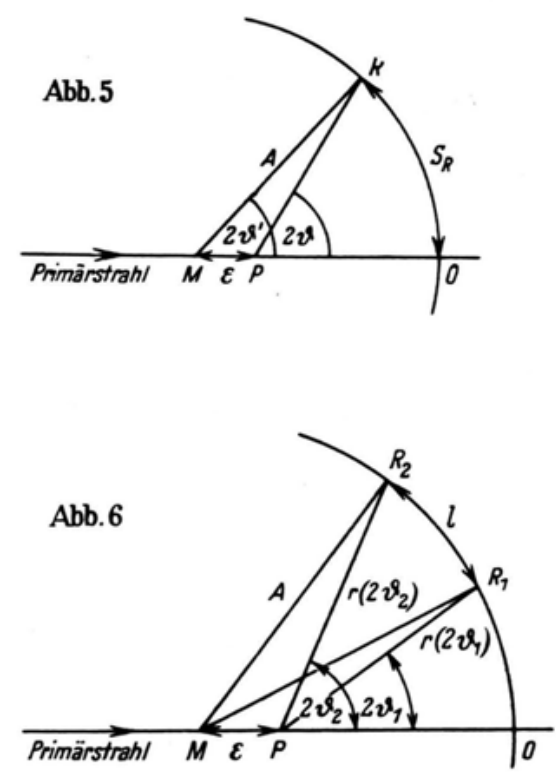

Abb. 5. Präparatexzentrizität $\varepsilon$ bei gegebenem Nullpunkt. Abb. 6. Präparatexzentrizität $\varepsilon$ bei fehlendem Nullpunkt.

zwei Punkten der Streukurve, festgelegt durch eine Eichaufnahme, bekannt ist. Für den Abstand $r$ einer unter dem Winkel $2 \vartheta$ auf dem Film auftretenden Interferenz vom Streuzentrum $\mathrm{P}$, das um die Strecke $\varepsilon$ gegen den Kammermittelpunkt $M$ versetzt sei, gilt nach Abb. 6

$$
r(2 \vartheta)=-\varepsilon \cos 2 \vartheta+\sqrt{A^{2}-\varepsilon^{2} \sin ^{2} 2 \vartheta},
$$

dabei ist $\varepsilon$ positiv in Richtung des Primärstrahls zu rechnen. Ist $\varepsilon \ll A$, so erhält man für den Abstand $l$ zweier unter den Streuwinkeln $2 \vartheta_{1}$ und $2 \vartheta_{2}$ auftretenden Interferenzen

$$
\begin{aligned}
l \approx & \int_{2}^{2 \vartheta_{2}} r(2 \vartheta) \mathrm{d} 2 \vartheta \\
& =\int_{2}^{2 \vartheta_{2}}\left(-\varepsilon \cos 2 \vartheta+\sqrt{A^{2}-\varepsilon^{2} \sin ^{2} 2 \vartheta}\right) \mathrm{d} 2 \vartheta .
\end{aligned}
$$


Entwickelt man die Wurzel in eine binomische Reihe, so wird

$$
\begin{array}{r}
l \approx A \int_{2 \vartheta_{1}}^{2 \vartheta_{2}}\left[-\frac{\varepsilon}{A} \cos 2 \vartheta+1-\left(\frac{\varepsilon}{A}\right)^{2} \frac{\sin ^{2} 2 \vartheta}{2}\right. \\
\left.-\left(\frac{\varepsilon}{A}\right)^{4} \frac{\sin ^{4} 2 \vartheta}{8}-\cdots\right] \mathrm{d} 2 \vartheta .
\end{array}
$$

Da die Exzentrizität $\varepsilon$ als klein gegen den Kammerradius $A$ angenommen ist, können die in $(\varepsilon / A)$ quadratischen Glieder und erst recht die höheren Potenzen von $(\varepsilon / A)$ vernachlässigt werden. Damit ergibt sich für den Abstand der betrachteten Interferenzen

$$
l \approx-\varepsilon\left(\sin 2 \vartheta_{2}-\sin 2 \vartheta_{1}\right)+A\left(2 \vartheta_{2}-2 \vartheta_{1}\right)
$$

und für die Exzentrizität

$$
\varepsilon \approx \frac{A\left(2 \vartheta_{2}-2 \vartheta_{1}\right)-l}{\sin 2 \vartheta_{2}-\sin 2 \vartheta_{1}} .
$$

Mit $\varepsilon$ folgt aus Abb. 6

oder

$$
\operatorname{tg} 2 \vartheta=\frac{r \sin s / r}{r \cos s / r-\varepsilon}
$$

$$
\frac{\sin \vartheta}{\lambda}=\frac{1}{\lambda} \sin \frac{1}{2} \operatorname{arctg} \frac{r \sin s / r}{r \cos s / r-\varepsilon} .
$$

Damit ist die gesuchte Beziehung $\sin \vartheta / \lambda=f(s)$ gefunden, die man zur Korrektion der Streukurve braucht.

Der Fall 2 läßt sich auf 1 zurückführen, wenn es gelingt, den Durchstoßpunkt O noch nachträglich festzulegen. Der Ausdruck $I_{\mathrm{R}} / I_{\mathrm{D}}$ (vgl. unten) ist eine mit $2 \vartheta$ monoton ansteigende Funktion; hierbei ist mit $I_{\mathrm{R}}$ bzw. $I_{\mathrm{D}}$ die Streuintensität der Reflexions- bzw. Durchstrahlungsseite für eine bestimmte Richtung $2 \vartheta$ bezeichnet. Trägt man $I_{\mathrm{R}}$ und $I_{\mathrm{D}}$ als Funktion des Abstandes $s$ von einem zunächst willkürlich gewählten Punkt auf, so müssen auch die experimentell bestimmten $I_{\mathrm{R}} / I_{\mathrm{D}}$-Werte monoton mit $s$ bzw. $2 \vartheta$ anwachsen, wenn der angenommene Bezugspunkt mit dem Nullpunkt identisch ist. Wurden durch die Wahl des Bezugspunktes den entsprechenden Kurvenpunkten der Reflexions- und Durchstrahlungsseite verschiedene $\sin \vartheta / \lambda$-Werte zugeordnet, dann treten im Kurvenverlaufe von $I_{\mathrm{R}} / I_{\mathrm{D}}$ Oszillationen auf. In solchem Falle sind die Streukurven der Reflexions- und Durchstrahlungsseite zu verschieben, bis diese Oszillationen verschwinden, d. h. bis der gewählte Bezugspunkt mit dem tatsächlichen Nullpunkt O identisch ist. Daneben bietet dieses Verfahren die Möglichkeit, den Einstellwinkel $\alpha$ auch für Aufnahmen festzulegen, bei denen sich $\alpha$ nicht aus dem Schattenbild bestimmen läßt. Hier liefert der Schnittpunkt der $I_{\mathrm{R}} / I_{\mathrm{D}}$-Kurve mit der Abszissenachse den gesuchten Einstrahlwinkel $\alpha=2 \vartheta$.

\section{Polarisationskorrektion}

Diese Korrektion berücksichtigt die bei der Monochromatisierung der Strahlung am Kristall entstehende Polarisation des Primärstrahles. Sie ist nach v a n d e r $\mathrm{G}$ rinte $\mathrm{n}^{14}$ gegeben durch

$$
P=\frac{1+\cos ^{2} 2 \varphi \cos ^{2} 2 \vartheta}{1+\cos ^{2} 2 \varphi},
$$

wo $\varphi$ den Glanzwinkel der reflektierenden Netzebene bedeutet.

\section{Absorptionskorrektion}

Durch die Absorptionskorrektion wird die im Präparat auftretende Schwächung der Strahlung eliminiert. Sie ist eine Funktion des Einstellwinkels $\alpha$, der Richtung der Streustrahlung $2 \vartheta$ und der Größe $\mu D$ des Präparates. Man erhält nach $\mathrm{Richter}$ und $\mathrm{F}$ ü r s t ${ }^{2}$ als Korrektionsfaktoren für die

$$
\begin{aligned}
& \text { Reflexionsseite } Q_{\mathrm{R}} \\
& =\frac{\sin (2 \vartheta-a)}{\sin a+\sin (2 \vartheta-a)}\left\{1-e^{-\mu D\left(\frac{1}{\sin a}+\frac{1}{\sin (2 \vartheta-a)}\right)}\right\},
\end{aligned}
$$

Durchstrahlungsseite $Q_{\mathrm{D}}$

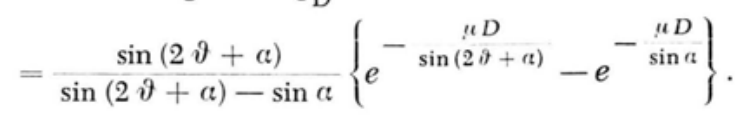

Die Berechnung der Absorptionskorrektionen setzt die Kenntnis des Einstellwinkels $\alpha$ und der Größe $\mu D$ voraus. Wie schon oben erwähnt, kann der Einstrahlwinkel $\alpha$ in erster Näherung unmittelbar der Aufnahme selbst entnommen werden. Damit ist die Möglichkeit gegeben, aus dem experimentell erhaltenen Intensitätsverhältnis $I_{\mathrm{R}} / I_{\mathrm{D}}$ die Größe $\mu D$ der untersuchten Schicht zu berechnen. Aus $c I_{0}=I_{\mathrm{R}} / Q_{\mathrm{R}}=$ $I_{\mathrm{D}} / Q_{\mathrm{D}}$, wo $c=c(2 \vartheta)$ den Bruchteil der in Richtung $2 \vartheta$ gestreuten Strahlung bedeutet, folgt

$$
\text { - } \frac{I_{\mathrm{R}}}{I_{\mathrm{D}}}=\frac{Q_{\mathrm{R}}}{Q_{\mathrm{D}}}=\frac{\sin (2 \vartheta-a)\{\sin (2 \vartheta+a)-\sin a\}\left\{1-e^{-\mu D\left(\frac{1}{\sin a}+\frac{1}{\sin (2 \vartheta-a)}\right)}\right\}}{\sin (2 \vartheta+a)\{\sin (2 \vartheta-a)+\sin a\}\left\{e^{-\frac{\mu D}{\sin (2 \vartheta+a)}}-e^{-\frac{\mu D}{\sin a}}\right\}} .
$$

14 W. van der Grinten, Physik. Z. 34, 609 [1933]. 


\begin{tabular}{|l|l|l|l|l|l|l|l|l|}
\hline \multicolumn{1}{|c|}{$\sin \vartheta / \lambda$} & 0 & 0,1 & 0,2 & 0,3 & 0,4 & 0,5 & 0,6 & \multicolumn{1}{c|}{0,7} \\
\hline Compton & 0 & 6 & 11 & 16 & 20 & 22 & 24 & 26 \\
Heisenberg-Bewilogua & 0 & 5,8 & 9,5 & 12,2 & 14,7 & 16,1 & 18,1 & 19,6 \\
Breit-Dirac-Faktor & 1,00 & 0,998 & 0,992 & 0,981 & 0,965 & 0,947 & 0,924 & - \\
\hline
\end{tabular}

Tab. 1. Inkohärente Streuintensität von As.

Die Bestimmung der Größe $\mu D$ und des genaueren Wertes von $\alpha$ kann mit Hilfe der experimentell gefundenen $\left(I_{\mathrm{R}} / I_{\mathrm{D}}\right)$-Werte durch sukzessive Näherung durchgeführt werden. Diese Methode hat den Vorteil, daß die der Aufnahme tatsächlich zugrunde liegenden Werte von $\alpha$ und $\mu D$ ohne weitere Messungen bestimmt werden können.

4. Korrektion auf inkohärente $\mathrm{S}$ trahlung

Hierzu berechnet man nach $\mathrm{H}$ e is e $\mathrm{n}$ be $\mathrm{rg}{ }^{15}$ und B e w i log u a ${ }^{16}$ die inkohärente Strahlung $I_{\text {ink }}$, welche die Atomanordnung des zu untersuchenden Stoffes im Beugungsdiagramm nicht abbildet. Die Werte $I_{\text {ink }}$ von As sind in Tab. 1 in absoluten Einheiten des Atomformfaktors $F^{2}$ für verschiedene $\sin \vartheta / \lambda$ angegeben, ebenso auch die Werte des Breit-Dirac-Faktors ${ }^{17}$ für $\mathrm{Cu}-\mathrm{K}_{a}-$ Strahlung, der die relativistische Korrektion der Compton-Streuung darstellt. Die Berücksichtigung dieser Korrektionen erfolgt im Zuge des Überganges zur absoluten Intensitätsskala.

5. Úbergang zu absoluten Intensitäten

Die bisher in willkürlichen Einheiten gemessenen Intensitäten sind zur Durchführung der Fourier-Analyse in den absoluten Einheiten von $F^{2}$ auszudrücken. Hierzu addiert man die berechnete inkohärente Streuintensität zu der kohärenten Atomstreuung $F^{2}$ und gleicht die korrigierte experimentelle Streukurve $I_{\text {korr }}$ der abgeänderten Atomformfaktorkurve $F^{2}+I_{\text {ink }}$ an. Notwendige Bedingung für eine solche Angleichung ist, daß die in den gesamten Raum ausgestrahlte Intensität von $N$ freien Atomen $N \int_{0}^{\pi}\left(F^{2}+I_{\text {ink }}\right) \sin \vartheta \mathrm{d} \vartheta$ gleich der Streuintensität von $N$ Atomen mit bestimmten Phasenbeziehungen zueinander sein muß. Bezeichnet man den Normierungsfaktor mit $c$, so muß mit B e r ckhem e r ${ }^{4}$ gelten

15 W. H e is e n berg, Physik. Z. 32, 737 [1931].

16 L. B e wilog u a, Physik. Z. 32, 740 [1931]; 33, 688 [1932].

17 Dieser Korrektionsfaktor ist sowohl eine Funktion von $\vartheta$ als auch von $\lambda$ allein, er erweist sich also nicht als Funktion des sonst üblichen Argumentes $\sin \vartheta / \lambda$.

$$
\int_{0}^{\pi}\left(F^{2}(\vartheta)+I_{\text {ink }}\right) \sin \vartheta \mathrm{d} \vartheta=c \int_{0}^{\pi} I(\vartheta)_{\text {korr }} \sin \vartheta \mathrm{d} \vartheta
$$

Die Intensitätswerte sind im Falle der Angleichung mit dem konstanten Faktor

$$
c=\frac{\int_{0}^{\pi}\left(F^{2}+I_{\mathrm{ink}}\right) \sin \vartheta \mathrm{d} \vartheta}{\int_{0}^{\pi} I_{\mathrm{korr}} \sin \vartheta \mathrm{d} \vartheta}
$$

zu multiplizieren, d. h. es ist $c I_{\text {korr }}=I_{\text {abs }}^{\prime}$. Den Normierungsfaktor $c$ gewinnt man durch graphische Auswertung der beiden Integrale. Da sowohl $I_{k o r r}$ als auch $F^{2}$ und $I_{\text {ink }}$ als Funktionen von $\sin \vartheta / \lambda$ gegeben sind, ist es für die Auswertung zweckmäßig, die Größe $\sin \vartheta / \lambda$ als Integrationsvariable einzuführen. Man erhält dann für den Normierungsfaktor

$$
c=\frac{\int_{0}^{1 / \lambda}\left(F^{2}+I_{\text {ink }}\right) \frac{\sin \vartheta}{i} \mathrm{~d} \frac{\sin \vartheta}{\lambda}}{\int_{0}^{1 / \lambda} I_{\text {kotr }} \frac{\sin \vartheta}{\lambda} \mathrm{d} \frac{\sin \vartheta}{\lambda}} .
$$

Als Kontrolle für eine solche Angleichung kann die Tatsache dienen, daß die so angeglichene Intensitätskurve $I_{\text {abs }}^{\prime}$ bei großen $\sin \vartheta / \lambda$-Werten in die $\left(F^{2}+I_{\text {ink }}\right)$-Kurve einmünden muß. Nach Normierung der korrigierten Streukurve $I_{\text {korr }}$ ist der inkohärente Streuanteil in Abzug zu bringen.

Um den Einfluß der Angleichung auf das Ergebnis der Fourier-Analyse zu prüfen, wurde schon bei den ersten Untersuchungen über das amorphe As (G e i ling und $\mathrm{Richter}{ }^{3}$ ), also bei Fehlen der obigen Angleichungsmethode, die Intensitätskurve $I_{\text {abs }}^{\prime}$ absichtlich so normiert, daß sie für große $\sin \vartheta / \lambda$-Werte in einem Falle oberhalb und im anderen unterhalb $\operatorname{der}\left(F^{2}+I_{\text {ink }}\right)$-Kurve verlief. Hierbei ergab sich, daß eine nicht genaue Angleichung die Atomabstände kaum, die Atomzahlen dagegen merklich beeinflußt.

Die weitere Auswertung der normierten Streukurve $I_{\mathrm{abs}}=I_{\mathrm{abs}}^{\prime}-I_{\mathrm{ink}}$ erfolgt nach Zernicke und 
Prins ${ }^{18}$, Debye und Menke ${ }^{10}$ sowie Warren und $\mathrm{G}$ ing ri $\mathrm{ch}^{19}$ mittels der Fundamentalgleichungen

$$
4 \pi r^{2} \varrho(r)=4 \pi r^{2} \varphi_{0}+\frac{2 r}{\tau} \int_{0}^{\infty} s \cdot i(s) \cdot \sin s r \cdot \mathrm{d} s
$$

und

$$
W(r)=\frac{g(r)}{\underline{g}_{0}},
$$

hierin bedeutet:

$$
\varrho_{0}=\text { mittlere Atomzahldichte }=\frac{L \cdot \sigma \cdot 10^{-24}}{A}
$$

(Atomzahldichte = Zahl der Atome in der Volumeneinheit $1 \AA^{3}, \sigma=$ Dichte, $L=$ Loschmidtsche Zahl, $A=$ Atomgewicht),

$\varrho(r)=$ Atomzahldichte im Abstande $r$ vom Ausgangsatom,

$s=4 \pi \sin \vartheta / i$.

$i(s)=\frac{I_{\mathrm{abs}}}{F^{2}}-1$.

\section{Ergebnis se}

1. Periodenwertevon amorphem Arsen

Die untersuchten As-Präparate lieferten im wesentlichen das gleiche Streubild. In allen Fällen handelt es sich um amorphes As, wie Geiling und Rich t e ${ }^{3}$ eingehend gezeigt haben. Abb. $7^{*}$ gibt das Streubild von gefälltem As wieder. Die Aufnahme zeigt das für Flüssigkeiten und feste amorphe Stoffe charakteristische Aussehen. Auffallend ist die endliche Schwärzung um den Nullpunkt, die für das Vorliegen sehr kleiner Teilchen spricht. Diese kontinuierliche Schwärzung trat nur beim chemisch gefällten As auf und vor allem dann, wenn bei tiefer Temperatur gefällt wurde. Weiter ist das Auftreten einer Interferenz bei kleinen Streuwinkeln mit stark wechselnder Lage (vgl. Tab. 2) überraschend. Diese innerste Interferenz, im folgenden als Vormaximum bezeichnet, wurde bei allen As-Präparaten beobachtet.

In Abb. 8 ist die korrigierte Intensitätskurve von gefälltem As mit angeglichener Atomformfaktorkurve wiedergegeben. In allen Fällen erhielt man für Präparate gleicher Herstellungsart dieselbe Kurve. Die Aufnahmen der chemisch gefällten Präparate, die aus Lösungen verschiedener Konzentration bei totaler und partieller Reduktion und verschiedenen Fällungstemperaturen erhalten wurden, stimmten ebenfalls weitgehend miteinander überein. Weder eine Änderung der Lage der Maxima noch eine wesentliche Änderung der Intensitätsverhältnisse der Streukurven

* Abb. 7, s. Tafel S. 724 b.

18 F. Zernike u. J. A. Prins, Z. Physik 41, 184 [1927]. konnte beobachtet werden. Das sublimierte As zeigt dagegen eine hiervon etwas abweichende Intensitätsverteilung.

Im Laufe der Untersuchungen ergab sich, daß es zur Bestimmung der Atomverteilung nicht von Vorteil ist, die sonst übliche Methode der Mittelwertsbildung auf die korrigierten Streukurven anzuwenden. Mitunter zeigten nämlich die Streukurven besonders für große $\sin \vartheta / \lambda$ zunächst unwesentlich erscheinende $\mathrm{Ab}$ weichungen im Intensitätsverlauf. Die Fourier-Analyse dieser Streukurven ergab dagegen zum Teil erhebliche Unterschiede im Aussehen der zugehörigen $W(r)$ Kurven. Diese Feinheiten der Atomanordnung, im

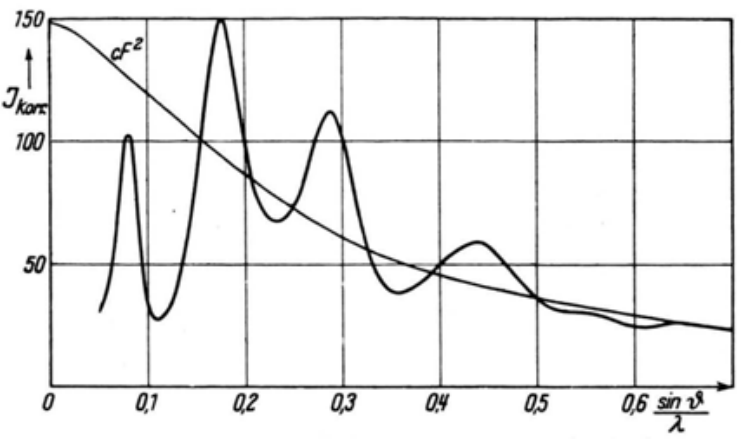

Abb. 8. Korrigierte Intensitätskurve von gefälltem As mit angeglichener Atomformfaktorkurve $\left(\mathrm{SnCl}_{2}=\right.$ Fällung).

Aufbau der amorphen Phase, gehen bei der Mittelwertsbildung verloren.

In Tab. 2 sind die $d$-Werte für die beobachteten Interferenzen der untersuchten As-Präparate zusammengestellt. Weiter sind die entsprechenden Werte von Geiling und Richter ${ }^{3}$ sowie Richter ${ }^{20}$ angegeben. Diese Zusammenstellung zeigt, daß bei den neuen Messungen das sublimierte As in der Lage des ersten Maximums, ganz besonders aber in der des Vormaximums, von den bei gefälltem As erhaltenen Werten abweicht.

Bei einem Vergleich mit den entsprechenden Werten von Geiling und Richter ${ }^{3}$ besteht für das mit $\mathrm{SnCl}_{2}$ gefällte As keine Übereinstimmung. In beiden Arbeiten werden im Fall des sublimerten As stets die kleinsten Periodenwerte erhalten. Während bei Geiling und Richter ${ }^{3}$ alle drei Streukurven deutliche Unterschiede aufweisen, zeigen die erneuten Versuche im Fall der beiden chemisch gefällten Präparate volle Übereinstimmung, und zwar sowohl im Hinblick auf die Lage als auch auf die Intensität der

19 B. E. W arren u. N. S. Ging r i ch, Physic. Rev 46, 368 [1934].

20 H. R i chter, Physik. Z. 44, 416 [1943]. 


\begin{tabular}{|c|c|c|c|c|c|}
\hline Herstellungsart & $\begin{array}{l}\text { Präp.- } \\
\text { Nr. }\end{array}$ & $d_{0}$ & $d_{1}$ & $d_{2}$ & $d_{3}$ \\
\hline $\begin{array}{l}\text { As sublimiert, } \\
\text { Schichtträger auf } \\
\text { Zimmertemperatur }\end{array}$ & $\begin{array}{l}\text { I } \\
\text { II } \\
\text { III } \\
\text { IV }\end{array}$ & $\begin{array}{l}5,40 \\
5,44 \\
5,37 \\
5,44\end{array}$ & $\begin{array}{l}2,74 \\
2,73 \\
2,75 \\
2,74\end{array}$ & $\begin{array}{l}1,74 \\
1,72 \\
1,72 \\
1,73\end{array}$ & $\begin{array}{l}1,13 \\
1,12 \\
1,12 \\
1,14\end{array}$ \\
\hline $\begin{array}{l}\text { As sublimiert, } \\
\text { Schichtträger } \\
\text { auf der Tem- } \\
\text { peratur der } \\
\text { flüssigen Luft }\end{array}$ & $\begin{array}{l}\text { V } \\
\text { VI } \\
\text { VII }\end{array}$ & $\begin{array}{l}5,05 \\
5,00 \\
5,10\end{array}$ & $\begin{array}{l}2,74 \\
2,73 \\
2,74\end{array}$ & $\begin{array}{l}1,73 \\
1,72 \\
1,73\end{array}$ & $\begin{array}{l}1,13 \\
1,12 \\
1,12\end{array}$ \\
\hline $\left.\begin{array}{c}\text { As gefällt mit } \mathrm{H}_{3} \mathrm{PO}_{2} \\
\text { bei Zimmertemp. }\end{array}\right\}$ & VIII & 6,20 & 2,86 & 1,74 & 1,14 \\
\hline $\left.\begin{array}{c}\text { As gefällt mit } \mathrm{SnCl}_{2} \\
\text { bei Zimmertemp. }\end{array}\right\}$ & IX & 6,25 & 2,86 & 1,74 & 1,14 \\
\hline $\left.\begin{array}{l}\text { As gefällt mit } \mathrm{H}_{3} \mathrm{PO}_{2} \\
\text { bei }-15^{\circ} \mathrm{C}\end{array}\right\}$ & $\mathrm{X}$ & 5,96 & 2,82 & 1,73 & 1,13 \\
\hline $\begin{array}{l}\text { As gefällt mit } \mathrm{SnCl}_{2} \\
\text { bei }-40^{\circ} \mathrm{C}\end{array}$ & XI & 6,10 & 2,80 & 1,74 & 1,15 \\
\hline \multicolumn{6}{|c|}{ Geiling und Richter } \\
\hline As sublimiert & XII & 5,20 & 2,72 & 1,71 & 1,10 \\
\hline As gefällt mit $\mathrm{SnCl}_{2}$ & XIII & 5,80 & 2,98 & 1,83 & 1,18 \\
\hline As gefällt mit $\mathrm{NaH}_{2} \mathrm{PO}_{2}$ & XIV & 5,80 & 2,83 & 1,73 & 1,14 \\
\hline $\left.\begin{array}{l}\text { As sublimiert (Elek- } \\
\text { tronenbeugung nach } \\
\text { Richter) }\end{array}\right\}$ & XV & $\begin{array}{c}5,13 \\
-6,15\end{array}$ & 2,76 & 1,74 & 1,13 \\
\hline
\end{tabular}

Tab. 2. Periodenwerte von amorphem As.

Interferenzen. Die hiervon verschiedenen Ergebnisse des sublimierten As hängen offensichtlich mit der Herstellungsart dieser Schichten, also mit der Kondensation aus der Dampfphase, zusammen.

\section{Atomabstände und Atomzahlen von} amorphem Arsen

Die aus der korrigierten Intensitätskurve durch Fourier-Analyse gewonnene radiale Dichteverteilung ist in Abb. 9 für die amorphe Aufdampfschicht des Präparates I wiedergegeben; sie zeigt die für nichtkristalline Stoffe charakteristische Nahordnung der Atome. Hinsichtlich der Analyse der Streukurve sei bemerkt, daß hier die Beachtung einiger praktischer Hinweise sehr von Vorteil ist. So wurden z. B. von der zu analysierenden si $(s)$-Kurve Schablonen hergestellt. Hierdurch wird die statistische Streuung der Punktlagen praktisch ausgeschaltet, so daß sich auch gewisse Feinheiten im Verlaufe der $W(r)$-Kurve abzeichnen können. Weiter wurde u. a. die Punktfolge äußerst dicht

21 L. R. Maxwell, S. B. Hendricks u. V. M. Mos le y, J. chem. Physics 3, 699 [1935].

22 Hier sind immer Schichten von etwa gleicher Dicke miteinander $\mathrm{zu}$ vergleichen. gewählt, ganz besonders an den kritischen Stellen. Im allgemeinen wurden mehr als 130 Punkte von jeder $W(r)$-Kurve durch die Fourier-Analyse festgelegt.

In Tab. 3 sind die der $4 \pi r^{2} \varrho(r)$-Kurve entnommenen Atomabstände $r_{v}$, (einschließlich der Kopplungsperioden), die Atomzahlen $n_{v}$ und die Kantenwinkel $\varphi$ für das kristalline und amorphe As zusammengestellt sowie einige Angaben über die Präparatherstellung gemacht. In dieser Tabelle sind für jedes Präparat zwei Zeilen bestimmt. Die obere Zeile enthält die beobachteten Atomabstände innerhalb der Einzelkette und die untere die eventuell auftretenden Perioden der Ketten- und Schichtkopplung. Da im sublimierten und gefällten (amorphen) As die 2. Koordination des Gitters fehlt, wird eine unterschiedliche Bezeichnungsweise für $r_{v}$ und $n_{v}$, bezüglich beider Zustände notwendig. So beziehen sich mit einem Stern versehene Größen $r_{v}{ }^{*}$ und $n_{v}{ }^{*}$ auf das Gitter. Tab. 3 zeigt, daß der Abstandswert $r_{1}$ und die Atomzahl $n_{1}$ im Gitter, in der amorphen Phase und im Molekül des As-Dampfes ( $\mathrm{M}$ axwell und Mitarbeiter ${ }^{21}$ ) näherungsweise dieselben sind. Die 2. Koordination des Gitters mit $r_{2}{ }^{*}=3,15 \AA$ tritt dagegen im amorphen As nicht auf. Der Abstandswert $r_{3}{ }^{*}=3,77 \AA$ ist bei allen As-Präparaten als bevorzugter Atomabstand wiederum vorhanden. Weiter geht aus Tab. 3 hervor, daß die Werte von $r_{1}$ und $r_{2}$ zum Teil erheblich kleiner als im Gitter sind. Die beobachteten Abstandswerte $r_{1}$ bis $r_{4}$ für das sublimierte ${ }^{22}$ und gefällte As stimmen unter sich recht gut überein, was vielleicht auf einen unterschiedlichen Aufbau der beiden As-Präparate hindeutet.

Für das amorphe As erhält man in Übereinstimmung mit dem Gitter in der 1. Koordination 3 Atome; dabei ergab sich für das sublimierte As ein etwas gröBerer und für das gefällte ein zum Teil etwas kleinerer Zahlenwert. In der 2. Koordination findet man etwa 9 Atome, und zwar sowohl beim sublimierten als auch beim gefällten As.

Streng genommen ist die exakte Bestimmung der Atomzahlen nur für die 1. Koordination möglich, da schon die 2. und ganz besonders die höheren Koordinationen stark ineinandergreifen, so daß eine Aufteilung in einzelne Atomgruppen nur schwer durchzuführen ist. Um eine schärfere Trennung der einzelnen Atomgruppen zu erreichen, ist es von Vorteil, die Aufdampfschichten bei tiefer Temperatur herzustellen und sie anschließend zu untersuchen; ähnlich wirkt bei der Fourier-Analyse auch die Ausdehnung des Integrationsbereiches nach großen $s$-Werten. Während die höheren Koordinationen, also die groBen Abstandswerte, bereits durch die Interferenzen 


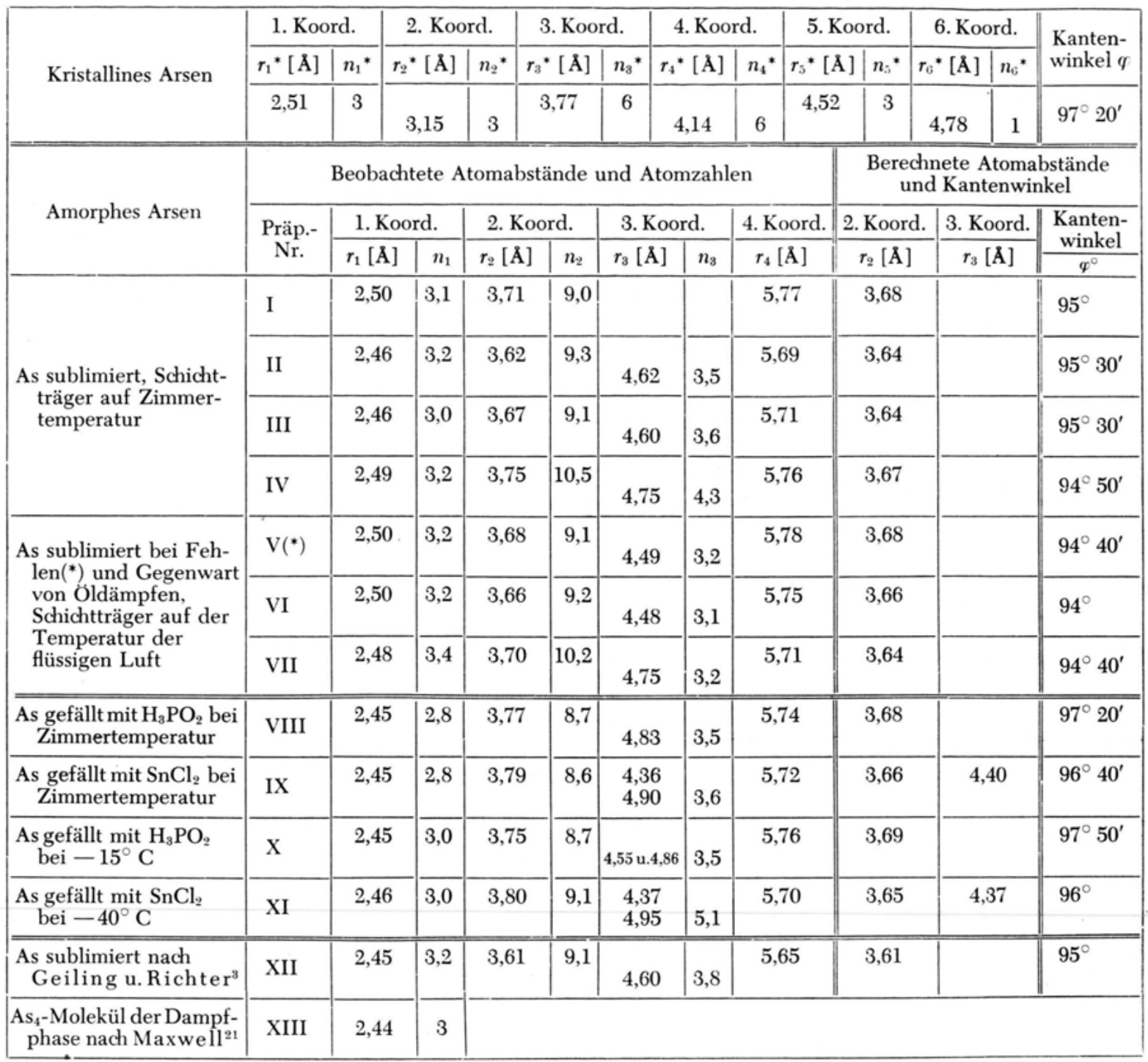

Tab. 3. Atomabstände, Atomzahlen und Tetraederwinkel in kristallinem und amorphem As nach der $4 \pi r^{2} \varrho(r)$-Kurve. Obere Zeile $=$ Atomabstände innerhalb der Kette. Untere Zeile = Atomabstände von Kette zu Kette bzw. von Schicht zu Schicht (Kopplungsabstände).

bei kleinen $\sin \vartheta / \lambda$ richtig erfaßt werden, erfordern die niedrigen Koordinationen die Ausdehnung des Streubildes zu möglichst hohen Werten von $\sin \vartheta / \lambda$.

Die Maxima der $4 \pi r^{2} \varrho(r)$-Kurve (Massenverteilungskurve) sind identisch mit den Stellen größter Massenbelegung; sie bestimmen die Lage der Atome und damit den Atomabstand. Die höheren Maxima sind in der $4 \pi r^{2} \varrho(r)$-Kurve weniger deutlich ausgeprägt, so daß hier die Bestimmung des Atomabstandes unmöglich ist. In solchem Falle geht man zur zugehörigen Dichteverteilungskurve über, bezogen auf die Dichte bei gleichmäßiger Massenverteilung; sie gibt die Häufigkeit der Atomlagen für verschiedene Abstandswerte $r$ vom betrachteten Zentralatom an (vgl. Abb. 10). Diese sog. W (r)-Kurve (Kurve für die Lagenwahrscheinlichkeit der Atome) läßt die Lage der Häufigkeitsmaxima auch für große $r$-Werte deutlich erkennen; sie ist daher zur Diskussion der bevorzugten Atomabstände geeigneter als die $4 \pi r^{2} \varrho(r)$ Kurve. Hierbei ist allerdings zu beachten, daß beim Úbergang zur $W(r)$-Kurve (Division der $4 \pi r^{2} \varrho(r)$ Kurve mit $4 \pi r^{2} \varrho_{0}$ ) die Maxima nach kleinen $r$-Werten hin verschoben werden. Die aus der $4 \pi r^{2} o(r)$-Kurve abgelesenen (bevorzugten) $r$-Werte sind daher allein 
geignet für einen unmittelbaren Vergleich mit den entsprechenden Abstandsgrößen des Gitters.

In den Abb. $11^{*}$ bis 13 sind einige elektronenmikroskopische Aufnahmen von sublimiertem und gefälltem As wiedergegeben. In Abb. 11 handelt es sich um eine Aufnahme von gefälltem As. Die Durchstrahlung ganzer Bezirke, insbesondere die feingegliederte Struktur ihrer Randzonen deutet das Vorliegen sehr kleiner Teilchen an. Im Gegensatz hierzu zeigen die Kügel-

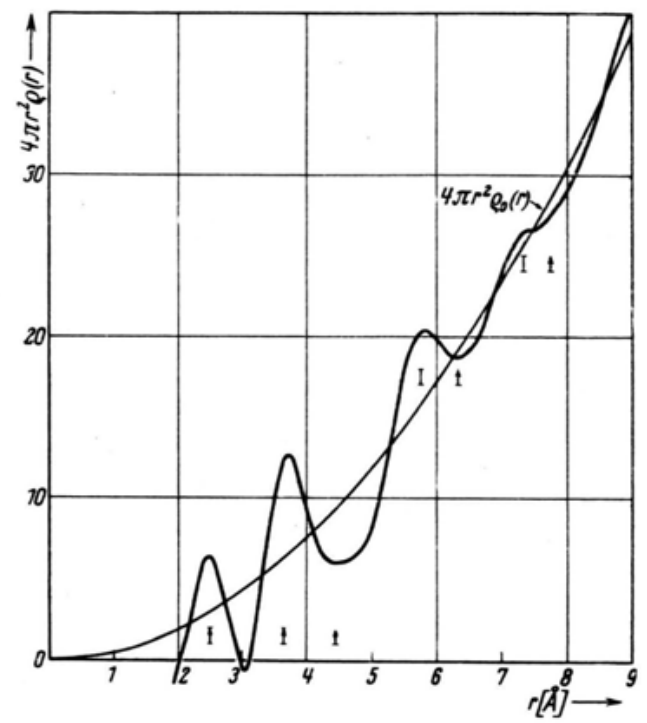

Abb. 9. Radiale Dichteverteilung in sublimiertem As (Präp. I: sehr dünne, mattschwarze Aufdampfschicht, $\left.D \approx 10^{-5} \mathrm{~cm}\right)$.

chen des sublimierten As in Abb. 12 keinerlei Struktur, d. h. hier sind die einzelnen Teilchen erheblich größer. Die zugehörige Aufnahme von umgewandeltem As (amorph $\rightarrow$ kristallin, $T_{\mathrm{U}}=285^{\circ} \mathrm{C}$, vgl. Abb. 13) läßt in den großen, kantigen Kristalliten die Kristallbildung zweifelsfrei erkennen.

V. Modellmäßige Deutung der Struktur des amorphen Arsens

Die Untersuchungen von $\mathrm{Z}$ a c harias e ${ }^{23}$ sowie Warren, Krutter und Morningstar ${ }^{24}$ an Gläsern $\left(\mathrm{B}_{2} \mathrm{O}_{3}\right.$ und $\left.\mathrm{SiO}_{2}\right)$ und neuerdings von $\mathrm{F}$ ürst, Glocker und Richter ${ }^{1}$ sowie $\mathrm{K}_{\text {önig }}{ }^{25}$ an Ge haben dazu geführt, gewisse Bausteine des Gitters auch als Baueinheiten für den amorphen Körper an-

* Abb. 11-13, s. Tafel S. 724 b.

23 W. H. Z a charias en, J. chem. Physics 3, 158 [1935]; J. Amer. ceram. Soc. 54, 3841 [1932].

24 B. E. W arren, H. Krut ter u. O. Morning st a r, J. Amer. ceram. Soc. 19, 202 [1936]. zunehmen. Im folgenden soll versucht werden, an Hand der neuen Ergebnisse den Aufbau des amorphen As durch die Existenz einer solchen Baueinheit des Gitters zu erklären.

1. Baustein oder Baugruppe ${ }^{26}$ des ArsenGitters als Bauelement des amorphen Zustandes

Die Kurve der Atomverteilung für eine sehr dünne mattschwarze Aufdampfschicht von As zeigt deutlich ausgeprägte Maxima bis zu großen Abstandswerten (vgl. W(r)-Kurve des Präparates I in Abb. 10). Von den Atomen werden also bestimmte Abstände be-

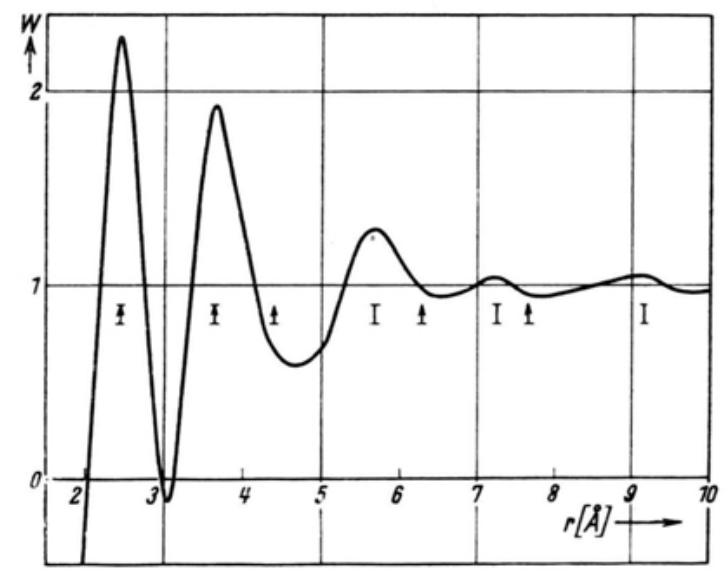

Abb. 10. Atomverteilung in sublimiertem As (Präp. I: sehr dünne, mattschwarze Aufdampfschicht, $D \approx 10^{-5} \mathrm{~cm}$ ).

vorzugt eingenommen. Weiter ersieht man aus Tab. 3, daß die Abszisse des ersten und zweiten Maximum 3 praktisch übereinstimmt mit dem Atomabstand in der 1. und 3. Koordination des As-Gitters, also mit der Länge der Seiten- und Basisflächenkante des Bauelementes des Gitters, eines einfachen gleichschenkligen Tetraeders $\left(\right.$ Kantenwinkel $=97^{\circ} 20^{\prime}$ ). Es kehren also die Abmessungen des Bauelementes des AsGitters im amorphen Zustand wieder, d. h. der Gitterbaustein ist im amorphen As erhalten geblieben. Die Kantenlänge der Gitterzelle $a_{\mathrm{Rh}}=4,14 \AA$ zeichnet sich dagegen in der $W(r)$-Kurve der Abb. 10 nicht ab, d. h. im amorphen As ist nicht mehr die Elementarzelle des As-Gitters vorhanden. Die normale Raumgitterstruktur von As ist also verlorengegangen. Ebenso tritt die 2. Koordination des Gitters $r_{2}{ }^{*}=3,15 \AA$ in

25 H. K ö n ig, Optik 3, 201 [1948].

26 Mit Baustein oder Bauelement ist der Gitterbaustein (As 4 -Tetraeder) und mit Baugruppe eine gesetzmäßige Verkettung von Gitterbausteinen bezeichnet. 
der $W(r)$-Kurve nicht in Erscheinung; im Gegenteil, dieser Abstandswert fällt in das ausgeprägte Minimum zwischen dem ersten und zweiten Maximum, d. h. hier besteht keine Möglichkeit, Atome anzutreffen. Um das Auftreten der übrigen bevorzugten Atomabstände zu verstehen, ist es notwendig, den Aufbau des As-Gitters kurz zu betrachten.

Dieses Gitter kann man in einzelne Schichten senkrecht zur Raumdiagonale der rhomboedrischen Elementarzelle zerlegen (vgl. Abb. 14); man kann es wie das Graphit-Gitter als Schichtengitter auffassen. Jedes Atom einer Schicht hat 3 nächste Nachbarn (1. Koordination), und zwar in der gleichen Schicht, der es selbst angehört. Die 3 Atome der 2. Koordination liegen in

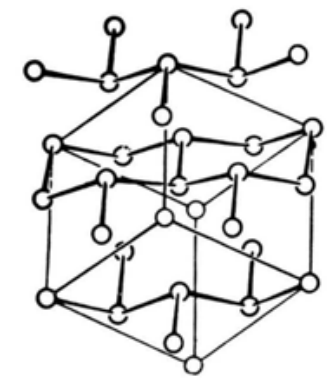

Abb. 14. Schichtstruktur von As. Aus „Röntgenanalyse von Krystallen" von J. M. B i j v o et, N. H. K olkm e i j e r u. M a c Gill avry (J. Springer, Berlin 1940).

der benachbarten Schicht und die 6 Atome der 3. Koordination wieder in der Ausgangsschicht. Diese innerlich stark gebundenen Schichten folgen im Gitter mit schwachen Bindungskräften aufeinander. Da im amorphen As die 2. Koordination des Gitters fehlt, muß hier die Bindung von Schicht zu Schicht offenbar verlorengegangen sein. Die Schichten des As-Gitters kann man wiederum in einzelne räumliche Ketten zerlegen, wenn man deren Bindungen längs der dunklen Stege in Abb. 15 löst, die von der eingezeichneten Geraden geschnitten werden. In Abb. 16 ist eine solche AsKette dargestellt. Diese Kette kann man sich ihrerseits durch eine gesetzmäßige Verknüpfung des Bausteines des As-Gitters aufgebaut denken. Die Verknüpfung der Elementar-Tetraeder geht unmittelbar aus Abb. 16 hervor. Danach hat jedes Tetraeder mit seinen benachbarten jeweils eine Kante, einen Schenkel, gemeinsam. Bei kontinuierlich fortschreitender geradliniger Verknüpfung der Tetraeder erhält man in der 1. Koordination, also im Abstand der Tetraederkante, genau wie im Gitter 3 Atome und in der 2. Koordination 4 Atome im Abstande der Basisflächenkante des Tetraeders (3. Koordination des As-Gitters!). Im fol- genden seien die mit geraden Zahlen gekennzeichneten Atome, die sich gegenseitig binden, deren Valenzen also abgesättigt sind, als die „Innenatome" der Kette bezeichnet und die ungeradzahlig benannten Atome sinngemäß als „Außenatome“ oder besser als „Randatome“.

Läge in den dünnen, mattschwarzen Aufdampfschichten von As eine räumliche Anordnung solcher regellos miteinander verknüpfter Ketten vor, dann müßte die $W(r)$-Kurve der Abb. 10 sämtliche Atom-

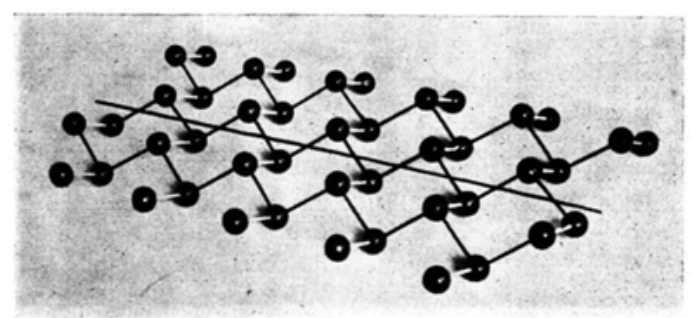

Abb. 15. Schichtebene des As-Gitters.

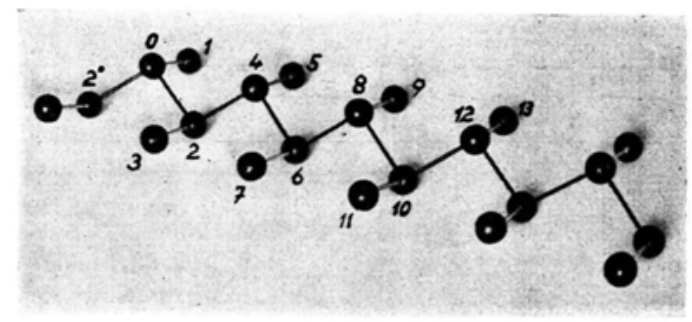

Abb. 16. Tetraederkette des As-Gitters.

abstände innerhalb der Kette liefern, d. h. alle Abstände zu den Innen- und zu den Randatomen, da in dieser Kettenanordnung andere Perioden nicht vorkommen. Zur Kettenbildung verknüpft man die Tetraeder zunächst mit den festen Abmessungen $r_{1}$ und $r_{2}$, wie in Abb. 16 geschehen. Dabei hat man bei jedem angeschlossenen Tetraeder noch die statistische Streuung der Atomlagen zu berücksichtigen, wie sie durch die Schwankungen der Abmessungen des Elementartetraeders, die Temperaturbewegung der Atome und nicht zuletzt durch eine partielle Drehung der miteinander verknüpften Tetraeder gegeben ist. Hierdurch werden die Entfernungen vom betrachteten Zentralatom des Ausgangstetraeders zu den Atomen benachbarter oder entfernt gelegener Tetraeder trotz streng gesetzmäßiger Verkettung immer undefinierter, da mit jedem neu angeschlossenen Tetraeder dieselbe Ungenauigkeit in seinen Abmessungen zu der bereits vorhandenen hinzutritt. Diese Ungenauigkeit der möglichen Abstandswerte muß zu einer Verflachung 


\begin{tabular}{|c|c|c|c|c|c|c|c|c|}
\hline Atomabstände & $r_{1}$ & $r_{2}$ & $r_{3}$ & $r_{4}$ & $r_{5}$ & $r_{6}$ & $r_{7}$ & $r_{8}$ \\
\hline $\begin{array}{c}\text { Beobachtet, Präparat I, } \\
\text { Kettenstruktur }\end{array}$ & 2,43 & 3,63 & & 5,67 & & 7,25 & & 9,15 \\
$\begin{array}{c}\text { Berechnet für Präparat I } \\
\text { nach dem Kettenmodell } \\
\text { Berechnet für die Kette } \\
\text { bzw. Schichtdes As-Gitters }\end{array}$ & 2,43 & 3,63 & 4,37 & 5,68 & 6,28 & 7,26 & 7,66 & 9,21 \\
\hline $\begin{array}{c}\text { Beobachtet, Präparat IX, } \\
\text { Schichtstruktur } \\
\text { Berechnet für Präparat IX } \\
\text { nach dem Ketten- bzw. } \\
\text { Schichtmodell }\end{array}$ & 2,40 & 3,77 & 4,52 & 5,89 & 6,52 & 7,54 & 7,95 & 9,57 \\
\hline
\end{tabular}

Tab. 4. Beobachtete und berechnete Atomabstände in der Kette bzw. Schicht bei As.

der Maxima in der $W(r)$-Kurve mit wachsendem $r$ führen, ohne daß dabei die Lage der Häufigkeitsmaxima merklich beeinflußt ${ }^{27}$ wird. Im vorliegenden Kettenmodell wird also die Bevorzugung gewisser Abstandswerte durch die gesetzmäßige Verkettung eines zunächst fest vorgegebenen Tetraeders und die Unschärfe der möglichen Atomabstände mit wachsendem $r$ durch die statistische Streuung der Atomlagen erreicht. Bei Verwendung der wahrscheinlichsten $r_{1^{-}}$ und $r_{2}$-Werte der Abb. 10, also diskreter Abstandswerte für alle Tetraeder, und bei streng gesetzmäßiger Verkettung müssen die berechneten Atomabstände in der einzelnen Kette der Abb. 16 mit den beobachteten Lagen der Häufigkeitsmaxima praktisch übereinstimmen, falls in den sehr dünnen, mattschwarzen Aufdampfschichten von As solche Ketten überhaupt vorkommen.

In den ersten drei Zeilen der Tab. 4 sind die Abszissenwerte der Häufigkeitsmaxima aus Abb. 10 und die berechneten Atomabstände für das Kettenmodell der Abb. 16 zusammengestellt, und zwar für das amorphe und kristalline As (Präp. I der Tab. 3 bzw. 6). Für die Abstandsberechnung sind die Abmessungen des Kettenbausteines als gegeben anzusehen; sie sind durch die Abszissenwerte des ersten und zweiten Maximums festgelegt. Tab. 4 zeigt, daß nur die Atomabstände für die Innenatome $(v=$ gerade) zu beobachten sind, und daß diese Abstandswerte mit den berechneten sehr gut übereinstimmen, und zwar bis zu den höchsten noch meßbaren Abständen. Es zeichnet sich also nur die aus den dunklen Stegen der Abb. 15 bzw. Abb. 16 gebildete Kette in $\operatorname{der} 4 \pi r^{2} \varrho(r)$ -

27 Eine begrenzte Drehung der angeschlossenen Tetraeder bringt für die Innenatome nur geringfügige Verlagerung der Häufigkeitsmaxima mit sich (vgl. Tab. 5). bzw. $W(r)$-Kurve ab. Die Abb. 9 und 10 geben diesen Sachverhalt anschaulich wieder. Hierin bezeichnen die Striche $T$ die berechneten Atomabstände innerhalb der Kette und die Pfeile $\hat{\imath}$ die Entfernungen zu den Randatomen; der Abstandsberechnung zugrunde gelegte (beobachtete) $r$-Werte sind durch einen PfeilStrich $\bar{\imath}$ markiert. Man sieht, daß die Maxima der $4 \pi r^{2} \varrho(r)$ - und der $W(r)$-Kurve mit den berechneten Abstandswerten für die Innenatome zusammenfallen und daß sich andererseits die Entfernungen zu den Randatomen keineswegs abzeichnen. Diese Übereinstimmung zwischen den experimentell beobachteten und den berechneten Atomabständen innerhalb der einzelnen Kette spricht für das Vorhandensein ganzer As-Ketten, also ganzer Baugruppen des Gitters im amorphen As. Wohl gemerkt, diese Ketten hat man sich nicht als „starre“, sondern als „elastische“ Gebilde vorzustellen; denn es sind die oben genannten statistischen Abweichungen der Atomlagen zuzulassen. Schon aus der Tatsache, daß der deutlich ausgeprägte Abstandswert $r_{4}$ bei den untersuchten AsProben mit den berechneten recht gut übereinstimmt (vgl. Tab. 4), ist zu schließen, daß diese räumlichen As-Ketten wie im Gitter vorwiegend gerade sind. Die Elementartetraeder sind also praktisch geradlinig miteinander zu verketten. Aus Abb. 10 entnimmt man als Mindestlänge für eine solche Kette $2 r_{\text {Min. }}=20,0 \AA$. So ist im amorphen As nicht nur der Gitterbaustein, sondern die ganze Kette des As-Gitters erhalten geblieben.

Wenn sich die Randatome in der W(r)-Kurve der Abb. 10 nicht abzeichnen, so ist das dahin zu deuten, daß die As-Ketten nicht frei, d. h. unabhängig voneinander, sondern nach den Ergebnissen der FourierAnalyse miteinander verkettet sind. Dabei werden 


\begin{tabular}{|c|c|c|c|c|}
\hline \multirow{2}{*}{$\begin{array}{c}\text { Atom- } \\
\text { Nr. }\end{array}$} & \multirow{2}{*}{$\begin{array}{l}\text { Atom- } \\
\text { abstände } \\
\text { in } \AA \\
\text { ohne } \\
\text { Drehung }\end{array}$} & \multicolumn{3}{|c|}{$\begin{array}{c}\text { Atomabstände in } \AA \\
\text { bei Drehung um }\end{array}$} \\
\hline & & $\varphi= \pm 10^{\circ}$ & $\varphi= \pm 20^{\circ}$ & $q= \pm 30^{\circ}$ \\
\hline $\begin{array}{l}0=\text { Zen- } \\
\text { tralatom }\end{array}$ & & & & \\
\hline 1 und 2 & 2,51 & & & \\
\hline 3 und 4 & 3,77 & & & \\
\hline 5 & 4,52 & $4,29 \rightarrow 4,74$ & $4,04 \rightarrow 4,97$ & $3,82 \rightarrow 5,17$ \\
\hline 6 & 5,89 & 5,89 & $5,84 \rightarrow 5,89$ & $5,76 \rightarrow 5,89$ \\
\hline 7 & 6,52 & $6,18 \rightarrow 6,83$ & $5,83 \rightarrow 7,13$ & $5,47 \rightarrow 7,28$ \\
\hline 8 & 7,54 & $7,49 \rightarrow 7,54$ & $7,35 \rightarrow 7,54$ & $7,11 \rightarrow 7,54$ \\
\hline
\end{tabular}

Tab. 5. Änderung der Atomabstände bei Drehung um die 2,4- und 4,6-Richtung der Abb. 16 für $\varphi= \pm 10^{\circ}, \pm 20^{\circ}$ und $\pm 30^{\circ}$.

offenbar die Randatome aus ihrer idealen Lage in statistisch regelloser Weise nach allen Richtungen abgebogen. Infolge dieser Unregelmäßigkeit der Atomlagen besitzen die Randatome keine ausgezeichneten Abstandswerte; sie zeichnen sich daher nicht in der $W(r)$-Kurve ab. Ihre Existenz ist jedoch durch die Atomzahl $n_{1}=3$ nachgewiesen (vgl. Tab.3). Der Ordnungsgrad in der As-Kette ist also nach der Seite weniger groß, so daß nur die Innenatome der Kette in der $W(r)$-Kurve in Erscheinung treten.

Diese Vorstellung vom verschwindenden Einfluß der Randatome hat kürzlich durch eine von G lo cke r erstmals bei Ge durchgeführte Rechnung einen anderen, offensichtlich mehr realen Sinn erhalten. Uberträgt man seine Úberlegungen auf die As-Kette der Abb. 16, so zeigt sich, daß bei begrenzter Drehung der angeschlossenen Tetraeder um die 2,4- und 4,6-Richtung als Achse die Atome innerhalb der Kette, also die Atome 6, 8 usf., kaum eine merkliche Abstandsänderung erfahren, ganz im Gegensatz zu den Randatomen 5, 7 usf. Die hierbei auftretenden Abstandsänderungen gibt Tab. 5 für die Kette des As-Gitters wieder. Danach durchlaufen die Abstandswerte der Atome 5, 7 usf. ein ganzes Längenintervall, das in der $W(r)$-Kurve lediglich einen diffusen Untergrund liefert. Die Abstände der Innenatome zeichnen sich dagegen diskret $a b$, da deren Änderungen verschwindend klein sind. Eine solche Drehung hat für sie nur eine geringfügige Verschiebung der Atomlagen und eine Verringerung der Abstandswerte zur Folge.

2. Kettenstruktur der sehr dünnen, mattschwarzen Aufdampfschichten von Arsen

Beim Sublimieren von As schlagen sich die Dämpfe in molekularer Form $\left(\mathrm{As}_{4}\right.$-Tetraeder) auf der Auf- fangfläche nieder. Betrachten wir ein solches einzelnes $\mathrm{As}_{4}$-Tetraeder, so schließen sich beim Aufdampfen an seine gleichberechtigten Kanten (Schenkel) neue Tetraeder an, d. h. es wachsen vom betrachteten Ausgangstetraeder gleichzeitig verschiedene As-Ketten in den Außenraum. An die Randatome dieser Ketten bauen sich durch Anlagerung neuer Tetraeder abermals weitere Ketten an. Dieser Vorgang der Kettenbildung pflanzt sich so lange fort, 'als es der freie, verfügbare Raum gestattet. Zwischen diesen Ketten sind offenbar Fremdgase eingelagert, ebenso sind die freien Valenzen abgebrochener Ketten zum Teil durch Fremdatome abgesättigt. In diesem räumlichen Gebilde miteinander verwachsener Ketten sind nach Abb. 10 die As-Ketten auf Längen von wenigstens $20 \AA$ praktisch gerade, d. h. es wird hier die geradlinige Struktur der Kette vorherrschend sein. Bei einer solchen Verknüpfung der Tetraeder können sich in der $W(r)$-Kurve neben einem kürzesten Abstand der Atome von Kette zu Kette (Kettenabstand) lediglich die Atomabstände innerhalb der Kette (Eigenstruktur der Kette) abzeichnen, da andere Perioden in dieser regellosen Kettenanordnung überhaupt nicht vorkommen. So zeigt die $W(r)$-Kurve der sehr dünnen, mattschwarzen Aufdampfschichten mit $D \approx 10^{-5} \mathrm{~cm}$ (vgl. Abb. 10) einmal sehr wenige Häufigkeitsmaxima, und zudem stimmt deren Lage mit den berechneten Atomabständen innerhalb der As-Kette überein, d. h. die Individualität der Kette spiegelt sich hier in der $W(r)$ Kurve in aller Deutlichkeit wieder. Wir haben es also in den sehr dünnen, mattschwarzen Aufdampfschichten von As mit einer regellosen, räumlichen Anordnung miteinander verknüpfter weitgehend gerader Ketten zu tun.

Für eine solche Verknüpfung der As-Ketten spricht vor allem die in der 2 . Koordination beobachtete Atomzahl $n_{2} \approx 9$ an Stelle von $n_{2}=4$ für die Einzelkette (vgl. Präparat I der Tab. 3). Die erste Gruppe der angeschlossenen Tetraeder liefert nämlich zwei weitere Atome für die 2.Koordination; die noch fehlenden Atome erbringt die regellose Lagerung der As-Ketten. Dabei nähern sich die Atome der Ketten auf einen bestimmten (charakteristischen) Abstandswert; für ihn gilt hier $r_{\text {Min. }} \approx r_{2}$.

3. Stäbchenstruktur der dicken Aufdampfschichtenvon Arsen

Mit wachsender Dicke der Aufdampfschicht $D>$ $10^{-4} \mathrm{~cm}$ bricht dieses räumliche Gebilde miteinander verbundener Ketten auf, und es tritt eine Parallelorientierung der As-Ketten ein. Die dicken Aufdampf- 
schichten zeigen dabei ein metallisch glänzendes Aussehen und die Stäbchenstruktur der Abb. 2. Das Vorhandensein solcher Stäbchen läßt unmittelbar auf eine Parallelorientierung der As-Ketten schließen. Wenn in diesen Aufdampfschichten eine solche Orientierung der As-Ketten vorliegt, so muß sich diese auch in der $W(r)$-Kurve abzeichnen. In diesem Falle müssen in ihr neue Perioden, Abstände der Atome von Kette zu Kette (Kopplungsperioden oder Kettenabstände), auftreten, die sich den Atomabständen innerhalb der Einzelkette überlagern. Abb. 17 zeigt die Verteilungskurve für die Atome einer solchen leicht metallisch

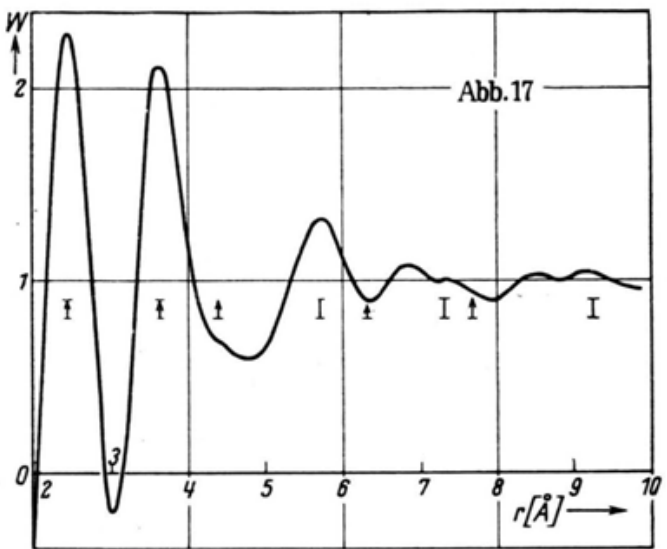

Abb. 17. Atomverteilung in sublimiertem As (Präp. V: dünne, tiefschwarze Aufdampfschicht, $D \geqq 10^{-4} \mathrm{~cm}$ ).

glänzenden Aufdampfschicht, die bei der Temperatur der flüssigen Luft hergestellt wurde. Ohne Kühlung der Auffangfläche sind die höheren Maxima der Kette und der Kopplung in der $W(r)$-Kurve längst nicht so scharf ausgeprägt. Die Kettenstruktur der Abb. 10 ist hier noch weitgehend erhalten geblieben, doch sind jetzt zwei neue Häufigkeitsmaxima bei $r=6,83 \AA$ und $r=8,45 \AA$ zu beobachten ${ }^{28}$. Das Auftreten dieser Abstandswerte ist offensichtlich durch eine Parallelorientierung der As-Ketten bei wachsender gegenseitiger Bindung zu erklären. Die neuen Atomabstände entsprechen demnach bestimmten Kopplungsperioden der Atome von Kette zu Kette. Bei stärkerem Aufdampfen geht die Eigenstruktur der Kette für große $r$-Werte immer mehr verloren, statt

28 Schichten mit ausgesprochener Stäbchenstruktur zeigen besonders deutlich die neue Periode $r=6,83 \AA$. Dieser Abstandswert zeichnet sich bereits bei Schichtdicken $D>10^{-4} \mathrm{~cm}$ ab. In Schichten dieser Dicke liegen also Stäbchen von submikroskopischer Längsausdehnung vor. Im folgenden wird daher der Begriff "Stäbchenstruktur“ auch auf solche dünne Schichten angewandt. dessen tritt, wie in Abb. $18 \mathrm{zu}$ beobachten ist, die neue Periode der Ketten-Kopplung $r=6,83 \AA$ um so stärker hervor. Die andere Kopplungsperiode $r=8,45 \AA$ ist dagegen von gewissen Ketten- bzw. Kopplungsabständen überlagert (vgl. Tab. 7b). Die scharf ausgeprägte Periode von Kette zu Kette $r=6,83 \AA$ stellt offenbar einen von den Atomen stark bevorzugten Abstandswert dar, so daß sich diese Periode in der $W(r)$-Kurve der Abb. 18 deutlicher abhebt als die großen Atomabstände innerhalb der Kette selbst. Dieser $r$-Wert ist sicherlich nicht die erste Kopplungs-

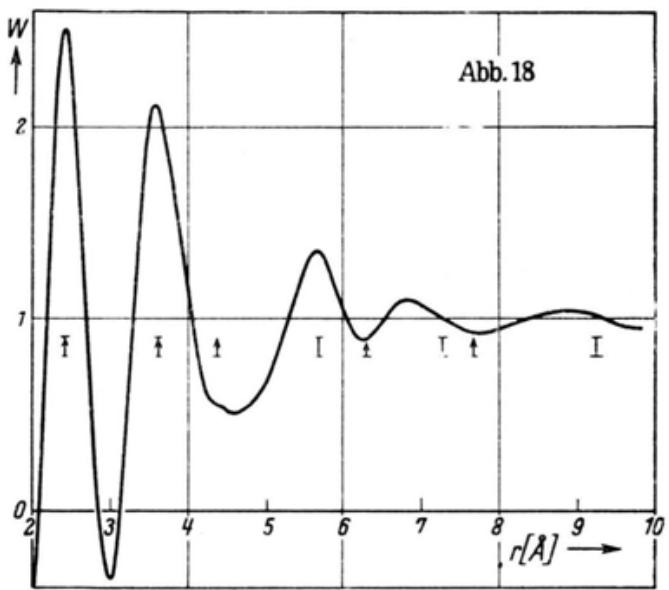

Abb. 18. Atomverteilung in sublimiertem As (Präp. III: dicke, metallisch glänzende Aufdampfschicht, $D>10^{-3} \mathrm{~cm}$ ).

periode für die As-Ketten. Kürzere Perioden dieser Art sind mit bestimmten Atomabständen der Kette identisch, bzw. sie liegen ganz in deren Nähe (vgl. Tab. 7 mit 3). Die zahlenmäßige Berechnung dieses Kettenabstandes $(r=6,83 \AA)$ aus der Parallelorientierung der Ketten würde die Vorstellung von der Stäbchenstruktur dicker Aufdampfschichten wesentlich stützen. In den Abb. 17 und 18 bestimmt für $r<6,0 \AA$ die Eigenstruktur der Kette und für $r>6,0 \AA$ in Abb. 17 die Einzelkette und ihre gegenseitige Orientierung und in Abb. 18 praktisch die Orientierung der Ketten den Verlauf der $W(r)$-Kurve. Die beobachteten Atomabstände innerhalb der Einzelkette und die Perioden der Ketten- bzw. Schichtkopplung sind in Tab. 6 zusammengestellt. Sämtliche Abstandswerte sind den $W(r)$-Kurven der untersuchten As-Proben entnommen. Bei den größten der hier beobachteten $r$-Werte ist infolge der U berlagerung von Ketten-, Stäbchen- oder Schichtstruktur eine Identifizierung der Periodenwerte mitunter nicht möglich. Diese Tabelle enthält ferner nähere Angaben über die Präparatherstellung, die 


\begin{tabular}{|c|c|c|c|c|c|c|c|c|c|c|c|}
\hline \multirow{2}{*}{\multicolumn{2}{|c|}{$\begin{array}{l}\text { Amorphes Arsen } \\
\text { (Präparatherstellung) }\end{array}$}} & \multirow{3}{*}{\begin{tabular}{|l}
$\begin{array}{c}\text { Präp.- } \\
\text { Nr. }\end{array}$ \\
I
\end{tabular}} & \multicolumn{8}{|c|}{ Atomabstände in $[\AA]$} & \multirow{3}{*}{$\mid \begin{array}{c}\begin{array}{c}\text { Strukturmodell } \\
\text { (Präparatstruktur) }\end{array} \\
\begin{array}{c}\text { Räumliche Anordnung } \\
\text { verknüpfter Ketten }\end{array}\end{array}$} \\
\hline & & & $r_{1}$ & $r_{2}$ & $r_{3}$ & $r_{4}$ & $r_{s}$ & $\boldsymbol{r}_{6}$ & $r_{\bar{z}}$ & $r_{8}$ & \\
\hline \multirow{4}{*}{$\begin{array}{l}\text { As sublimiert, } \\
\text { Schichtträger } \\
\text { auf Zimmer- } \\
\text { temperatur }\end{array}$} & $\begin{array}{l}\text { Viele sehr dünne Schichten } \\
\quad\left(D \approx 10^{-5} \mathrm{~cm}\right)\end{array}$ & & 2,43 & 3,63 & & 5,67 & & 7,25 & & 9,15 & \\
\hline & $\begin{array}{l}\text { Sehr dicke Schicht } \\
\qquad\left(D \approx 10^{-2} \mathrm{~cm}\right)\end{array}$ & II & 2,41 & 3,57 & 4,50 & 5,59 & & $\begin{array}{l}7,20 \\
6,85\end{array}$ & & 8,99 & $\begin{array}{l}\text { Feste Kopplung (Par- } \\
\text { allelorientierung) der } \\
\text { Ketten (Stäbchen- } \\
\text { struktur) }\end{array}$ \\
\hline & $\begin{array}{l}\text { Einzelne dicke Schichten } \\
\quad\left(D>10^{-3} \mathrm{~cm}\right)\end{array}$ & III & 2,42 & 3,62 & 4,52 & 5,65 & & $\begin{array}{l}7,30 \\
6,84\end{array}$ & & 8,97 & $\begin{array}{l}\text { Stäbchenstruktur: } \\
\text { Feste Kopplung }\end{array}$ \\
\hline & $\begin{array}{l}\text { Dünne Schicht auf Glas } \\
\left(\mathrm{D}<10^{-4} \mathrm{~cm}\right)\end{array}$ & IV & 2,44 & 3,68 & 4,70 & 5,65 & & 7,08 & & 8,90 & $\begin{array}{r}\text { Lose Kopplung der Ket- } \\
\text { ten (Stäbchenstruktur) }\end{array}$ \\
\hline \multirow{3}{*}{$\begin{array}{l}\text { As sublimiert } \\
\left.\text { bei Fehlen( }{ }^{*}\right) \text { u. } \\
\text { Gegenwart von } \\
\text { Öldämpfen, } \\
\text { Schichtträger } \\
\text { auf der Tem- } \\
\text { peratur der } \\
\text { flüssigen Luft }\end{array}$} & $\begin{array}{l}\text { Wenige dünne Schichten } \\
\quad\left(D \geqq 10^{-4} \mathrm{~cm}\right)\end{array}$ & $\mathrm{V}(*)$ & 2,45 & 3,64 & 4,40 & 5,70 & & $\begin{array}{l}7,30 \\
6,83\end{array}$ & 8,44 & 9,20 & $\begin{array}{l}\text { Stäbchenstruktur: } \\
\text { Feste Kopplung }\end{array}$ \\
\hline & $\begin{array}{l}\text { Viele dünne Schichten } \\
\quad\left(D \approx 10^{-4} \mathrm{~cm}\right)\end{array}$ & VI & 2,42 & 3,60 & 4,40 & 5,65 & & 7,00 & 8,55 & 9,22 & $\begin{array}{l}\text { Stäbchenstruktur: } \\
\text { Feste Kopplung }\end{array}$ \\
\hline & $\begin{array}{l}\text { Wenige dünne Schichten } \\
\quad\left(D \leqq 10^{-4} \mathrm{~cm}\right)\end{array}$ & VII & 2,42 & 3,65 & 4,70 & 5,65 & & 7,05 & 8,18 & 8,90 & $\begin{array}{l}\text { Stäbchenstruktur: } \\
\text { Lose Kopplung }\end{array}$ \\
\hline \multicolumn{2}{|c|}{ As gefällt mit $\mathrm{H}_{3} \mathrm{PO}_{2}$ bei Zimmertemp. } & VIII & 2,40 & 3,72 & 4,78 & 5,65 & & 7,05 & & 8,85 & $\begin{array}{l}\text { Stäbchenstruktur: } \\
\text { Lose Kopplung }\end{array}$ \\
\hline \multicolumn{2}{|c|}{ As gefällt mit $\mathrm{SnCl}_{2}$ bei Zimmertemp. } & IX & 2,40 & 3,76 & $\begin{array}{l}4,35 \\
4,79 \\
\end{array}$ & 5,64 & 6,40 & 7,14 & 7,85 & $\begin{array}{l}9,25 \\
8,80\end{array}$ & Schichtbildung \\
\hline \multicolumn{2}{|c|}{ As gefällt mit $\mathrm{H}_{3} \mathrm{PO}_{2}$ bei $-15^{\circ} \mathrm{C}$} & $\mathrm{X}$ & 2,41 & 3,70 & $\begin{array}{c}4,50 \\
\text { u. } 4,85\end{array}$ & 5,70 & 6,50 & 7,24 & 8,22 & 9,01 & Schichtbildung \\
\hline \multicolumn{2}{|c|}{ As gefällt mit $\mathrm{SnCl}_{2}$ bei $-40^{\circ} \mathrm{C}$} & XI & 2,42 & 3,74 & $\begin{array}{l}4,40 \\
4,90 \\
\end{array}$ & 5,67 & 6,40 & 7,19 & 8,08 & 8,92 & Schichtbildung \\
\hline \multicolumn{2}{|c|}{$\begin{array}{l}\text { As sublimiert, sehr dicke Schicht } \\
\left.\text { (Geiling und Richter }{ }^{3}\right)\end{array}$} & XII & 2,40 & 3,57 & 4,55 & 5,55 & & 6,83 & & 8,90 & $\begin{array}{l}\text { Stäbchenstruktur: } \\
\text { Feste Kopplung }\end{array}$ \\
\hline
\end{tabular}

Tab. 6. Beobachtete Atomabstände in amorphem As nach der $W(r)$-Kurve. Obere Zeile $=$ Atomabstände innerhalb der Kette. Untere Zeile = Kopplungsabstände.

Präparatdicke für die Einzelschicht und die vorliegende Präparatstruktur.

Betrachtet sei im Zusammenhang mit den Aufdampfschichten von As noch die $W(r)$-Kurve der Abb. 19; sie wurde von einer dünnen Aufdampfschicht auf Glas als Träger (Präparat IV, $D<10^{-4} \mathrm{~cm}$ ) erhalten. Aufallend ist hier, daß sich bei $r=4,70 \AA$ eine neue Periode abzuzeichnen beginnt und eine weitere bei $r=7,08 \AA$ auftritt. Die Diskussion dieser $W(r)$ Kurve, ebenso auch die zahlenmäßige Berechnung der Periode $r=6,83 \AA$ soll nach der Besprechung der Struktur des gefällten amorphen As erfolgen.

In den mattschwarzen Aufdampfschichten von As mit $D \approx 10^{-5} \mathrm{~cm}$ haben wir es also mit einer regellosen räumlichen Anordnung miteinander verknüpfter As-Ketten zu tun, in den Aufdampfschichten mit $D \geqq 10^{-4} \mathrm{~cm}$ dagegen mit einer Parallelorientierung der As-Ketten (Stäbchenstruktur). Im ersten Falle erhält man im wesentlichen die Perioden der einzelnen
Kette und im zweiten daneben noch die von Kette zu Kette.

4. Schichtbildung des chemisch gefällten Arsens

Etwa die gleiche $W(r)$-Kurve wie in Abb. 19 nur mit geringen Abweichungen in den Atomabständen erhält man bei dem chemisch gefällten As-Präparat VIII der Tab. 6. Auffallend ist hier genau wie bei dem Aufdampfpräparat IV (vgl. Abb. 19) das Auftreten einer neuen Periode bei etwa $r=4,78 \AA$. Dieser Abstandswert ist wie alle Atomabstände dieser Art (zweitnächster Schichtabstand) in Tab. 6 auf der unteren Zeile aufgeführt. Zur Diskussion dieser Periode betrachten wir in Abb. 20 die W(r)-Kurve des chemisch gefällten As-Präparates IX. Hier, wie überhaupt bei den amorphen As-Präparaten der $\mathrm{SnCl}_{2}$ Fällung, insbesondere der Tieftemperaturfällung, zeichnet sich diese neue Periode $(r=4,79 \AA)$ sehr 
deutlich ab, während sie bei den Sublimationsschichten höchstens andeutungsweise vorhanden ist. Die Größe dieses Periodenwertes differiert von Präparat zu Präparat. Uberraschenderweise treten hier an den Stellen, welche die Entfernungen zu den Randatomen festlegen, schwache Maxima auf; ihre berechneten Lagen sind durch Pfeile hervorgehoben. Offenbar haben sich im amorphen As der $\mathrm{SnCl}_{2}$-Fällung die Ketten bereits zu Schichten zusammengeschlossen, so daß jetzt auch die Randatome definierte Raumlagen einnehmen. Die geringe Höhe dieser Maxima und die beobachteten Atomzahlen (vgl. Tab. 3) sprechen für einen weniger großen Ordnungsgrad der Randatome

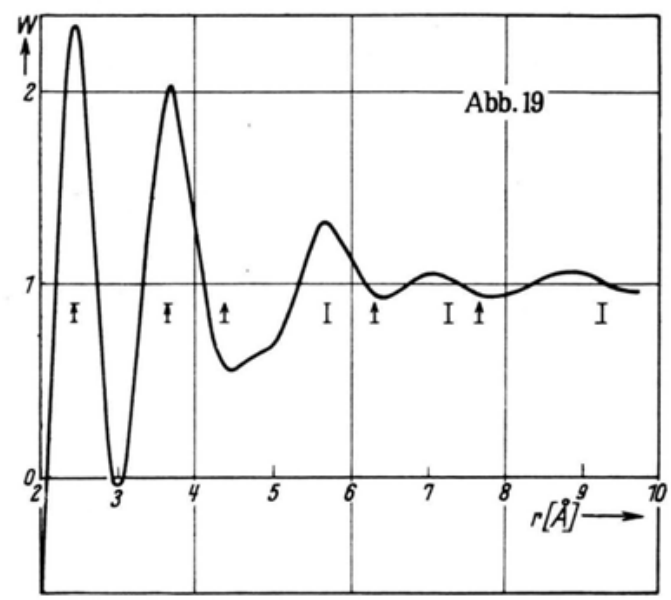

Abb. 19. Atomverteilung in sublimiertem As (Präp. IV: dünne, grauschwarze Aufdampfschicht, $D<10^{-4} \mathrm{~cm}$ ).

innerhalb der Schichten bzw. für das Vorliegen von Schichten mit geringen Querabmessungen. Ähnlich wie bei der $W(r)$-Kurve des Aufdampfpräparates IV ist auch hier die Periode innerhalb der Kette $r_{6}=2\left(r_{2}\right)_{\text {ber. }}=7,26 \AA$ nach kleineren $r$-Werten $(r=7,14 \AA)$ verlagert. Viel stärker fällt aber das Auftreten der neuen Periode $r=4,79 \AA$ auf; sie entspricht in Abb. 19 (Präparat IV, $D<10^{-4} \mathrm{~cm}$ ) der schwach angedeuteten Periode $r=4,70 \AA$. Da bei der vorliegenden Schichtbildung bezüglich der betrachteten $r$-Werte keine weiteren Atomabstände zu erwarten sind, als sie die Einzelkette (einschließlich der Randatome) selbst liefert, liegt es nahe, die neue Periode $r=4,79 \AA$ als Schichtabstand zu deuten und sie mit der Rhomboederkante des As-Gitters zu identifizieren. Im gefällten amorphen As liegt gemäß der hier beobachteten Schichtbildung eine dem Gitter weitgehend ähnliche Struktur vor. Für eine solche Schichtbildung sprechen auch die in Tab. 3 aufgeführten Kantenwinkel für das gefällte As.

Bei der Diskussion der $W(r)$-Kurven insbesondere des Zahlenmaterials der Tab. 6 sind eine ganze Reihe von Fragen offen geblieben; sie seien kurz zusammengestellt.

1. $\left(\bar{r}_{2}\right)_{\text {beob. }}=3,73 \AA$

für das gefällte As

$\left(\bar{r}_{2}\right)_{\text {beob. }}=3,57 \AA$

für das sublimierte As

(dicke Schicht)

Die Berechnung von $\left(r_{2}\right)_{\text {ber. }}=\sqrt{\frac{r_{4}{ }^{2}-r_{1}{ }^{2}}{2}}$ liefert:

$\left(r_{2}\right)_{\text {ber. }}=3,63 \AA$ bzw. $3,62 \AA$.

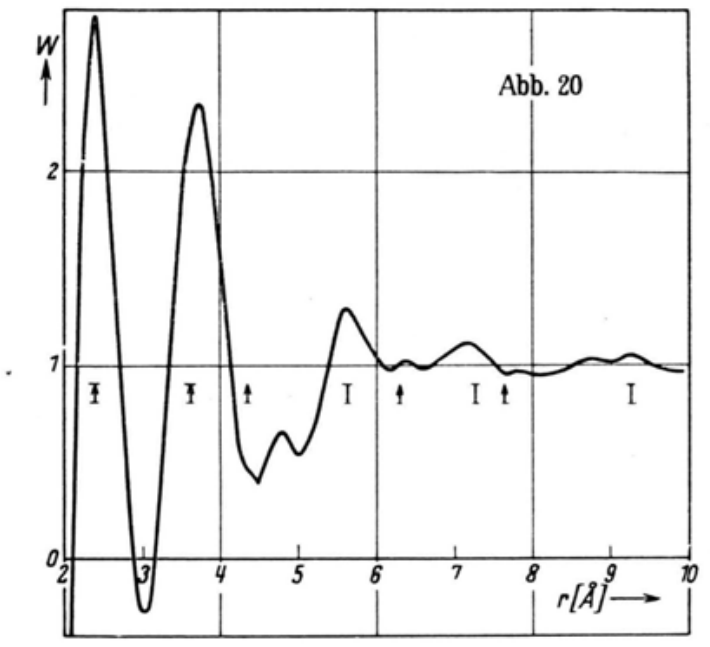

Abb. 20. Atomverteilung in gefälltem As (Präp. IX: schwarzes, pulverförmiges As).

2. Verlagerung von $r_{6}=7,26 \AA$ nach $r=7,14 \AA$ im gefällten As.

3. Berechnung der neuen Periode $r=6,83 \AA$ für das sublimierte As.

4. Erklärung des Dichteunterschiedes (etwa 20\%) von gefälltem und sublimiertem As.

Zu Punkt 1 sei bemerkt, daß man der Tab. 6 als Mittelwert für das gefällte As $\left(\bar{r}_{2}\right)_{\text {beob. }}=3,73 \AA$ entnimmt, während die Aufdampfpräparate II und XII (sehr dicke Schichten mit Stäbchenstruktur) den Wert $\left.\overline{(} r_{2}\right)_{\text {beob. }}=3,57 \AA$ liefern, der sich mit abnehmender Schichtdicke dem des gefällten As nähert. Diese Verlagerung des $r_{2}$-Wertes ist offenbar durch das Hineinspielen einer Kopplungsperiode verursacht. Berechnet man nach der oben angegebenen Beziehung den Atomabstand $r_{2}$ innerhalb der As-Kette bei gegebenem $r_{1}$ und $r_{4}\left(r_{4}=\right.$ Abszissenwert des dritten deutlich 
ausgeprägten Maximums der $W(r)$-Kurve), so erhält man nach Tab. 6 für das gefällte As $\left(\bar{r}_{2}\right)_{\text {ber. }}=3,63 \AA$ und für das sublimierte $\left(\bar{r}_{2}\right)_{\text {ber. }}=3,62 \AA$. Die gute Úbereinstimmung dieser Mittelwerte zeigt, daß der benützte $r_{4}$-Wert durch keine Kopplungsperiode merklich beeinflußt ist. Nach der gleichen Beziehung sind die $r_{2}$-Werte der Tab. $3\left(4 \pi r^{2} \varrho(r)=\right.$ Kurve!) berechnet.

Legt man in Tab. 4 (Präparat IX) für die Abstandsberechnung die Werte $r_{1}=2,40 \AA$ und $r_{2}=3,63 \AA$ zugrunde, so betragen bei gefälltem As die Abweichungen zwischen den beobachteten und den be-

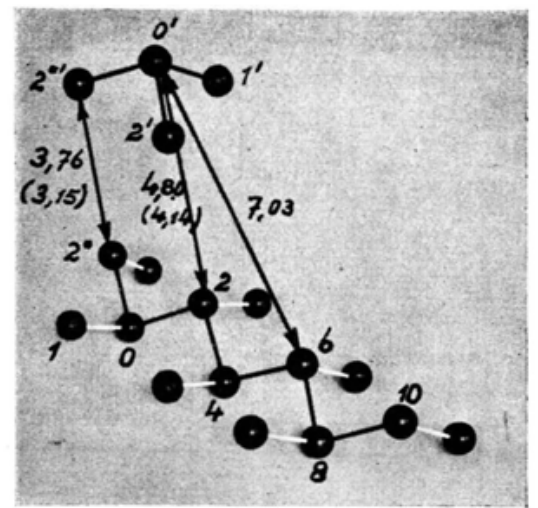

Abb. 21. Lagerung der As-Ketten bei Parallelorientierung [Zahlenwerte ( ) = entsprechende Gitterabstände].

rechneten Abstandswerten selbst für große Abstände weniger als $3 \%$ (vgl. auch Abb. 20).

Zur Berechnung der Kopplungsperiode, die zur Verlagerung des beobachteten $r_{2}$-Wertes führt, sei Abb. 21 betrachtet. Hier sind zwei übereinanderliegende Schichten des As-Gitters wiedergegeben, und zwar sind diese Schichten durch eine As-Kette bzw. ein einzelnes $\mathrm{As}_{4}$-Tetraeder dargestellt. Die Lage der Atome entspricht dabei bis auf die Randatome der im As-Gitter. Identifiziert man nach Abb. 21 die neue Periode $r=4,79 \AA$ mit dem Abstand der Atome 2 bzw. 2* vom Atom 0', so errechnet man in Anlehnung an das As-Gitter für den kürzesten Atomabstand $r_{2}{ }_{2}^{\prime}=r_{2}{ }^{*}{ }_{2}{ }^{*}=3,76 \AA$ sowie für den $\mathrm{Ab}$ stand $r_{0}{ }_{6}{ }_{6}=7,03 \AA$. Dabei übernimmt die neue, deutlich ausgeprägte (beobachtete) Periode $r=4,79 \AA=$ $r_{0}{ }_{2}^{\prime}$ die Rolle der Rhomboederkante $\left(r_{4}^{*}=a_{\mathrm{Rh}}=\right.$ $4,14 \AA$ ) und die kürzeste (berechnete) Ketten- bzw. Schichtdistanz $r_{2}^{\prime}{ }_{2}=r_{2}{ }^{\prime \prime}{ }_{2}{ }^{*}=r_{\text {Min. }}=3,76 \AA$ entspricht der 2. Koordination des As-Gitters $r_{2}{ }^{*}=3,15 \AA$ (Gitterdistanz). Der ebenfalls berechnete Abstandswert $r_{0}{ }^{\prime}=$
$7,03 \AA$ stellt einen häufig wiederkehrenden Abstand der Atome von Kette zu Kette dar. Die möglichen (kürzesten) Atomabstände für zwei unter $0^{\prime}$ gelagerten Ketten bzw. Schichten sind bei Zugrundelegung von $r_{1}=2,42 \AA, r_{2}=3,63 \AA$ und $r=4,79 \AA$ bzw. $r=4,55 \AA$ in Tab. 7 a bzw. b zusammengestellt. Die Entfernungen zu den Randatomen sind hierin nicht berücksichtigt. Häufig auftretende Abstandswerte sind in der mittleren Zeile aufgeführt; in der letzten Zeile sind die kürzesten Entfernungen zu den Atomen der übernächsten Kette angegeben. Man sieht, daß der kleinste Abstand der Atome von Schicht zu Schicht $\left(r_{\text {Min. }}\right)_{\text {sehicht }}=3,76 \AA$ beträgt. Bei der Stäbchenstruktur der Aufdampfschichten sind die Ketten ebenfalls parallel zueinander orientiert. Während sich bei der Schichtstruktur die Randatome weitgehend in die Schichtebene eingestellt haben, sind sie im Falle der Stäbchenstruktur wegen der geringfügigen Verdrehungen der angeschlossenen Kettenbausteine beliebig gegen die Vorzugsrichtung orientiert. Aus der bloßen Parallelität der Ketten folgt nach Abb. 21, wenn wir den Atomabstand $r_{0}{ }_{2}^{\prime}=r_{0}{ }_{2}{ }^{*}=4,55 \AA$ setzen, für die kürzeste Entfernung der Atome von Kette zu Kette $\left(r_{\text {Min. }}\right)_{\text {Kette }}=3,52 \AA$. Die beobachtete Periode $r=4,55 \AA$ stellt danach den zweitnächsten Kettenabstand dar.

Bei den chemisch gefällten Präparaten mit Schichtbildung hat man in der 2. Koordination: 6 Schichtatome im Abstande $r_{2}=3,63 \AA$ und 3 Atome in der benachbarten Schicht im (kürzesten) Abstande $\left(r_{\text {Min. }}\right)_{\text {Schicht }}=3,76 \AA$ also insgesamt 9 Atome; nach Tab. 3 in guter Úbereinstimmung mit dem experimentellen Befund. Der Schichtabstand $\left(r_{\text {Min. }}\right.$ ) schicht $=$ $3,76 \AA$ verschiebt den Abszissenwert des zweiten Häufigkeitsmaximums $\left(r_{2}=3,63 \AA\right)$ nach größeren $r$-Werten hin. Ebenso verlagert der Abstand $(r)_{\text {sehicht }}$ $=7,03 \AA$ die Periode innerhalb der Kette $r_{6}=7,26 \AA$ (vgl. Tab. 4) nach kleineren $r$-Werten, wie in den Abb. 19 und 20 zu beobachten ist. Bei den dicken As-Schichten des Aufdampfprozesses hat man dagegen in der 2. Kordination: 4 Atome innerhalb der betrachteten Kette im Abstande von $r_{2}=3,62 \AA$ und nach Tab. 3 etwa 5 Atome in den benachbart gelegenen Ketten im (kürzesten) Abstande von $\left(r_{\text {Min. }}\right)_{\text {Kette }}=3,52 \AA$, d. h. das zweite Maximum der $W(r)$-Kurve $\left(r_{2}=3,62 \AA\right)$ ist hier nach kleineren $r$-Werten verlagert.

In Tab. $7 \mathrm{~b}$ ist als ein häufig auftretender Kettenabstand u. a. auch der Wert $r=6,83 \AA$ aufgeführt (vgl. Abb. 21). Dieser charakteristische Atomabstand entspricht im gefällten As der Persiode $r=7,03 \AA$. 


\begin{tabular}{|c|c|c|c|c|c|c|}
\hline \multicolumn{4}{|c|}{ a) Gefälltes Arsen } & \multirow{2}{*}{\multicolumn{3}{|c|}{$\begin{array}{c}\text { b) Sublimiertes Arsen } \\
\text { Endatom }=()\end{array}$}} \\
\hline $\begin{array}{l}\text { Ausgangs- } \\
\text { atom }\end{array}$ & \multicolumn{3}{|c|}{ Endatom $=()$} & & & \\
\hline $\begin{array}{l}0^{\prime} \\
0^{\prime} \\
2^{\prime}\end{array}$ & $\begin{array}{l}\text { (0) }: 5,53 \\
(2): 4,79 \\
(2): 3,76\end{array}$ & $\begin{array}{l}\text { (4) }: 6,61 \\
\text { (6) }: 7,03 \\
(6): 5,23\end{array}$ & $\begin{array}{r}(8): 9,14 \\
(10): 10,00 \\
(10): 8,20\end{array}$ & $\begin{array}{l}\text { (0) }: 5,24 \\
(2): 4,55 \\
(2): 3,52\end{array}$ & $\begin{array}{l}\text { (4) }: 6,38 \\
(6): 6,83 \\
(6): 5,06\end{array}$ & $\begin{array}{r}(8): 8,98 \\
(10): 9,90 \\
(10): 8,09\end{array}$ \\
\hline \multicolumn{4}{|c|}{8,$30 ; 8,89$ und 10,06} & \multicolumn{3}{|c|}{$7,77,8,32$ und 9,37} \\
\hline
\end{tabular}

Tab. 7. Nächste Abstände der Atome für benachbarte Ketten bzw. Schichten.

Seine Berechnung ${ }^{29}$ erfolgt in gleicher Weise, also lediglich unter der Annahme einer Parallel-Lagerung der As-Ketten ähnlich wie im Gitter. Diese Identität des beobachteten und berechneten $r$-Wertes spricht ebenfalls für die Richtigkeit der hier dargelegten Vorstellungen vom Aufbau der dicken As-Schichten mit Stäbchenstruktur. Für große Abstandswerte hebt sich in den einzelnen $W(r)$-Kurven bald der eine, bald der andere Schicht- bzw. Kettenabstand der Tab. 7 etwas stärker ab, bzw. er bestimmt allein den weiteren Kurvenverlauf. Das Auftreten der neuen Perioden $r=4,79 \AA, r=7,03 \AA$ usf. bzw. $r=4,55 \AA, r=6,83 \AA$ usf. spricht also für eine Parallelorientierung der As-Ketten mit loser bzw. fester Packung (Stäbchenstruktur). Beim Präparat VIII ist ähnlich wie bei den Präparaten IV (vgl. Abb. 19) und VII diese neue Periode nur schwach ausgeprägt; auch zeichnen sich hier in der $W(r)$-Kurve selbst die nächsten Randatome nicht als Maxima ab. Aus dem Auftreten dieser nur schwach ausgebildeten Periode ist auf eine beginnende Parallel-Lagerung der AsKetten bei loser Packung zu schließen.

Im gefällten amorphen As liegt also eine gitterähnliche Struktur vor; sie zeigt bei gewisser Auflockerung etwa die gleichen Atomabstände innerhalb der einzelnen Schicht wie im Gitter, jedoch weit gröBere Abstandswerte für die Atome von Schicht zu Schicht. Im Gegensatz hierzu besitzen die Aufdampfschichten von As lediglich Ketten- oder Stäbchenstruktur oder beides nebeneinander. In den dicken Sublimationsschichten sind die Ketten enger aneinandergelagert als im gefällten As. Hierdurch erklärt sich die größere Dichte des sublimierten As. Eine losere Packung der As-Ketten beobachtet man allerdings auch in den sehr dünnen Aufdampfschichten

20 Für die entsprechenden Perioden errechnet man nach der $4 \pi r^{2} \varrho(r)$-Kurve mit $r_{1}=2,45 \AA, r_{2}=3,67 \AA$ und $r=4,90 \AA$ bzw. mit $r_{1}=2,49 \AA, r_{2}=3,66 \AA$ und $r=4,62 \AA$ für das gefällte bzw. sublimierte As die Werte $r=7,12 \AA$ bzw. $r=6,93 \AA$. (vgl. Präparate IV und VII der Tab. 6). Mit zunehmender Dicke der Sublimationsschicht lagern sich die Ketten offenbar enger aneinander, bis die Packungsdichte der dicken Schichten von Stäbchenstruktur erreicht ist. Mit dem in Tab. 6 aufgeführten Zahlenmaterial ist es im allgemeinen möglich, eine Aussage über die spezielle Form der nichtkristallinen Struktur der vorliegenden As-Probe zu machen.

In Tab. 6 fällt die gute Reproduzierbarkeit der beobachteten Periodenwerte auf, das gilt besonders für die chemisch gefällten Präparate. Diese As-Proben zeigten bei gleicher Herstellungsart weitgehend die gleiche Struktur (Schichtbildung), ganz im Gegensatz zu den Aufdampfschichten, deren Struktur (Kettenund Stäbchenstruktur) von Stelle zu Stelle mitunter stark variierte. Bei einem Vergleich der beobachteten $r_{1}$-Werte in Tab. 3 sieht man, daß die chemisch gefällten Präparate den kleinsten, die dicken AsProben mit Stäbchenstruktur praktisch denselben und die dünnsten Aufdampfschichten den größten $\mathrm{Ab}$ standswert $\left(r_{1} \approx r_{1}{ }^{*}\right)$ liefern. Ein Vergleich der Tab. 2 mit 3 zeigt weiter, daß aus einer Lageänderung der Interferenzen nicht auf eine entsprechende Abstandsänderung der Atome geschlossen werden darf.

Aus diesen Untersuchungen an As geht hervor, daß im amorphen Zustand ganze Baugruppen (gerade Ketten) des Gitters erhalten geblieben sind. Diese Ketten können, vielleicht unabhängig voneinander, also frei vorkommen, sie können aber auch miteinander verbunden, regellos (Abb. $10=$ räumliches $\mathrm{Ge}-$ bilde verknüpfter As-Ketten) oder geordnet (Abb. 18 $=$ Stäbchenstruktur) gelagert und schließlich durch Schichtbildung zu einer gitterähnlichen Struktur (Abb. 20 = Schichtbildung) zusammengeschlossen sein. In allen diesen Fällen liegt eine amorphe, nichtkristalline Phase von As mit mehr oder weniger gitterähnlichem Aussehen vor. Es sind also bei As verschiedene Grade des amorphen Zustandes zu unterscheiden. Im allgemeinen werden diese verschiedenen Formen des amorphen As nicht getrennt, sondern gleichzeitig 
nebeneinander beobachtet. Hierdurch wird die Deutung der erhaltenen $W(r)$-Kurven und damit die Vorstellung vom Aufbau der vorliegenden amorphen AsProbe sehr erschwert.

Der unterschiedliche Aufbau der As-Proben drückt sich auch in den elektronenmikroskopischen Aufnahmen aus, und zwar in einer Kugel- bzw. Teilchenstruktur. In der Aufdampfschicht der Abb. 12 (Kugelgestalt) liegt sicherlich eine räumliche Anordnung miteinander verknüpfter Ketten vor; im gefällten As der Abb. 11 (Teilchenstruktur) hat man es dagegen mit aufgelockerten As-Schichten von gitterähnlichem Aussehen zu tun.

VI. Ubergang: amorph $\rightarrow$ kristallin bei Arsen

Beim Sublimieren von As im Glas- oder Quarzrohr liefern die As-Proben, die vom innersten Rand der Sublimationsschicht stammen, Streubilder, die, visuell gesehen, ganz das Aussehen einer rein amorphen AsSchicht wie Abb. 7 zeigen. Die Fourier-Analyse solcher Aufdampfschichten läßt dagegen erkennen, daß teilweise Kristallisation stattgefunden hat. Da im kristallinen und amorphen Zustand das Bauelement das gleiche ist, ist hier das Auftreten von Atomabständen von Bedeutung, welche die Elementarzelle, die Struktureinheit des Raumgitters, festlegen und im amorphen As als charakteristische Gitterabstände naturgemäß fehlen. Bei einem Vergleich der $W(r)$ Kurve in Abb. 22 mit der von rein amorphem As fällt auf, daß der tiefe Einschnitt zwischen dem ersten und zweiten Maximum, der anfänglich bis unter die Abszissenachse reichte, jetzt zum großen Teil durch die Gitterdistanz $r_{2}{ }^{*}=3,15 \AA$ kompensiert ist. Noch wichtiger ist, daß sich auf dem abfallenden Kurvenzuge des zweiten Maximums in einer leichten Wellung bei $r_{4}{ }^{*}=a_{\mathrm{Rh}}=4,14 \AA$ die Rhomboederkante der Elementarzelle abzuzeichnen beginnt. Als weitere Größe der Gitterzelle von As sei der Abstandswert $r_{6}{ }^{*}=$ $4,78 \AA$ genannt, der die Entfernung vom unteren Eckatom bis zum Innenatom auf der Raumdiagonale mißt. Der Gitterabstand $r^{*}=5,78 \AA$ (Entfernung vom Innenatom bis zum oberen Eckatom der Elementarzelle) verlagert das dritte deutliche Maximum der $W(r)$-Kurve von amorphem As $\left(r_{4} \approx 5,66 \AA\right)$ praktisch nach diesem Gitterwert. Ebenso ist das zweite Maximum $\left(r_{2}=3,62 \AA\right)$ durch den Gitterabstand $a_{\mathrm{Rh}}=4,14 \AA$ erheblich nach großen $r$-Werten verschoben. Diese $W(r)$-Kurve zeigt deutlich, wie sich mit fortschreitender Kristallisation Atomabstände der Gitterzelle abzuzeichnen beginnen, d. h. es hat sich in einzelnen, allerdings sehr kleinen Bezirken der noch weitgehend amorphen Aufdampfschicht bereits das normale Raumgitter von As herausgebildet. Ob sich die Ketten der Aufdampfschichten von amorphem As beim Erhitzen auf die Umwandlungstemperatur $T_{\mathrm{U}}=285^{\circ} \mathrm{C}$ zunächst ebenfalls zu Schichten und damit zu einer gitterähnlichen Struktur zusammenschließen, wurde hier nicht untersucht. Fest steht, daß sich der nichtkristalline Zustand von As mit der beobachteten Schichtbildung in seinem Aufbau schon weitgehend dem As-Gitter genähert hat.

Hinsichtlich der Kristallisation amorpher Stoffe läßt sich ganz allgemein sagen, daß sich die Bausteine oder richtiger gesprochen die Baugruppen im amor-

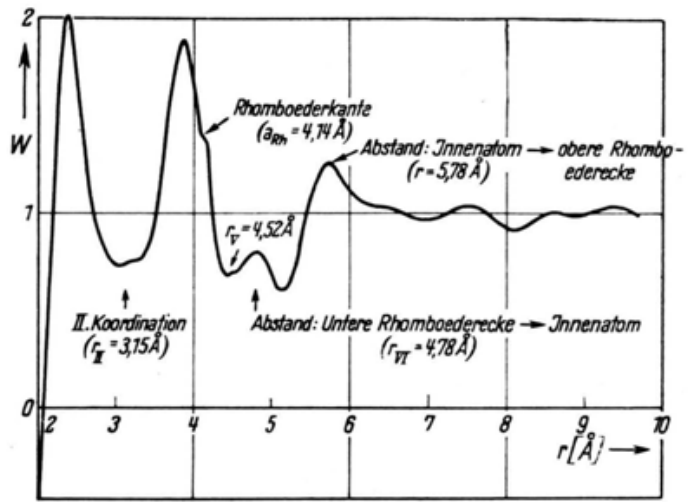

Abb. 22. Atomverteilung in einer teilweise umgewandelten Aufdampfschicht von As.

phen Körper beim Kristallisationsvorgang in die nach den Symmetriegesetzen des Gitters allein möglichen Lagen einstellen. Hierbei geht der amorphe Körper in den kristallinen Zustand über, und gleichzeitig werden eingeschlossene Gase frei, welche die Existenz der amorphen Phase ermöglichten und stabilisierten (vgl. Richter ${ }^{20}$ sowie Geiling und Richter ${ }^{3}$ ).

Bei der Deutung der Struktur amorpher Stoffe ist der nichtkristalline (glasige) Körper, mehr als bisher geschehen, in enger Anlehnung an das zugehörige Gitter zu betrachten. Der amorphe Zustand scheint nicht von allgemeiner Realisierbarkeit zu sein, vielmehr ist er stark von der Konstitution des Gitters abhängig. So wäre bei J mit seinem Molekülgitter im nichtkristallinen Zustand vielleicht ein Haufwerk einzelner Moleküle (eingefrorenes Gas) zu erwarten. Bei den Gittern mit Kettenstruktur wie $\mathrm{S}^{30}$, Se, weniger

30 Neben der Kettenform (Fadenschwefel) kommt der Schwefel auch als Molekülgitter mit $\mathrm{S}_{8}$-Ringen als Baueinheiten vor. 
bei Te, ist die amorphe Phase leicht zu verwirklichen, ebenso auch bei den Gittern mit Schichtbildung wie $\mathrm{P}$, As, Sb, weniger bei Bi. Beide Gittertypen sind durch die Gitterdistanz ausgezeichnet, d. h. durch eine schwache Bindung von Kette zu Kette bzw. von Schicht zu Schicht. Diese Bindung geht offenbar im amorphen $\mathrm{Se}$, As und $\mathrm{Sb}$ verloren. Hier wurden, wie erwartet, Ketten und auch Schichten beobachtet. Bei den übrigen Elementen liegt im Gitter eine gleichartige Atomanordnung in allen drei Raumrichtungen vor, so daß hier die Chance für die Existenz der amorphen Phase vielleicht weniger groß ist, es sei denn, daß im Gitter wie z. B. bei Si und Ge ganze
Atomgruppen (reguläre Tetraeder) als Baueinheiten auftreten. So ist auch bei $\mathrm{Si}$ und $\mathrm{Ge}$ der amorphe Zustand zu erreichen; hier liegt nach Richter und Breitling ${ }^{31}$ ebenfalls eine regellose räumliche Anordnung miteinander verknüpfter Ketten vor.

Herrn Prof. Dr. R. G l o c k e r danken wir herzlich für seine fördernden Ratschläge und sein stetes Interesse an dieser Arbeit, ebenso sei auch der Notgemeinschaft der Deutschen Wissenschaft für die Bereitstellung apparativer Hilfsmittel gedankt.

31 H. Richter u. G. B reitling, „Struktur des amorphen Germanium und Silicium." Die Arbeit erscheint in Kürze.

\title{
Die Randschicht eines Germanium-Flächengleichrichters im Flußbereich
}

\author{
Vorläufige Mitteilung \\ Von W. Bösenberg und E. Fues \\ Aus dem Institut für theoretische und angewandte Physik der Technischen Hochschule Stuttgart \\ (Z. Naturforschg. 6 a, 741-744 [1951]; eingegangen am 25. Juli 1951) \\ Erich Regener zum 70. Geburtstag \\ Einige Rechnungen, die über die Feld- und Konzentrationsverteilung der Elektronen und \\ Löcher in der S c h o t t k y schen Randschicht eines Metall/Germanium-Kontaktes durchgeführt \\ wurden, lassen den Schwemmeffekt in der Umgebung der Emittorelektrode eines Transistors \\ durch die S chottky sche Theorie verstehen. Bei großen Flußströmen ergibt sich im Halb- \\ leiter eine stark erhöhte Elektronen- und Löcherkonzentration.
}

I $\mathrm{m}$ Anschluß an eine Arbeit von van Roos broeck ${ }^{1}$, der die Feld- und Konzentrationsverteilung der Elektronen und Löcher im halbleitenden Germanium nach Löcherinjektion unter Berücksichtigung der Rekombination, aber unter Vernachlässigung der Raumladung berechnet hat, wurden diese Verteilungen für einen Metall/Germanium-Kontakt mit Inversionsschicht im Bereich der raumladungsbehafteten Schottky schen Randschicht unter Vernachlässigung der Rekombination berechnet. Die Lösungen der nicht-linearen Grundgleichungen [vgl. S chottky², Gln. $(5,10)$ und 5,11$)^{*}$ ] sind nicht geschlossen erhältlich. Genaue Lösungen wurden mit einem numerischen Iterations. verfahren nach v on $\mathrm{S}$ and e ${ }^{3}$ für den Strom Null und für einen großen Flußstrom bei Germanium mit

1 W. vain Roosbroeck, Bell System Techn. J. 29, 560 [1950].

2 W. S ch o t t k y u. E. S p e n k e, Wiss. Veröff. Siemens-Konzern 18, 225 [1939].

$3 \mathrm{H}$. von $\mathrm{S}$ anden, Praxis der Differentialgleichungen, W. de Gruyter, Berlin 1945.

4 W. S c hot tk y, Z. Physik 113, 367 [1939]. einer Störstellendichte von $10^{15} \mathrm{~cm}^{-3}$ berechnet. Nach $\mathrm{S} \mathrm{ch} \mathrm{ot} \mathrm{t} \mathrm{k} \mathrm{y}{ }^{4}$ sollte sich das thermische Gleichgewicht der Metallelektronen auch bei großen Flußströmen auf den Halbleiter auswirken und Anlaß zu stromunabhängigen Randwerten der Elektronen- und Löcherkonzentrationen - dies wurde als zulässige Randbedingung angesehen - geben. Die zugehörige elektrische Randfeldstärke wurde durch Probieren so bestimmt, daß sich von einem endlichen Wert der Ortskoordinate ab, nämlich am Ende der Randschicht, Raumladungsfreiheit ergibt, was weiterhin — immer unter Vernachlässigung der Rekombination - zu konstanten Werten der Elektronen- und Löcherdichte und zu einem konstanten Wert der Bahnfeldstärke führen würde. Die weitere Lösung sollte aber nach van Roosbroeck ${ }^{1}$ fortgesetzt werden.

* Die Gl. $(5,10)$ von 2 ist einmal für die Elektronenkonzentration $p$ und einmal für die Löcherkonzentration $q$ anzuschreiben. In $\mathrm{Gl} .(5,11)$ ist statt $f(p)$ die stromabhängige Differenz $q-p$ gegen die Werte für den Strom Null im raumladungsfreien Halbleiterinneren einzusetzen. 